\title{
The frequency of planetary debris around young white dwarfs ${ }^{\star}$
}

\author{
D. Koester ${ }^{1}$, B. T. Gänsicke ${ }^{2}$, and J. Farihi ${ }^{3}$
}

\author{
${ }^{1}$ Institut für Theoretische Physik und Astrophysik, Universität Kiel, 24098 Kiel, Germany \\ e-mail: koester@astrophysik.uni-kiel.de \\ 2 Department of Physics, University of Warwick, Coventry CV4 7AL, UK \\ 3 University College London, Department of Physics \& Astronomy, Gower Street, London WC1E 6BT, UK
}

Received 21 February 2014 / Accepted 18 April 2014

\begin{abstract}
Context. Heavy metals in the atmospheres of white dwarfs are thought in many cases to be accreted from a circumstellar debris disk, which was formed by the tidal disruption of a rocky planetary body within the Roche radius of the star. The abundance analysis of photospheric elements and conclusions about the chemical composition of the accreted matter are a new and promising method of studying the composition of extrasolar planetary systems. However, ground-based searches for metal-polluted white dwarfs that rely primarily on the detection of the Ca II K line become insensitive at $T_{\text {eff }}>15000 \mathrm{~K}$ because this ionization state depopulates.

Aims. We present the results of the first unbiased survey for metal pollution among hydrogen-atmosphere (DA type) white dwarfs with cooling ages in the range $20-200 \mathrm{Myr}$ and $17000 \mathrm{~K}<T_{\text {eff }}<27000 \mathrm{~K}$.

Methods. The sample was observed with the Cosmic Origins Spectrograph on board the Hubble Space Telescope in the far ultraviolet range between 1130 and $1435 \AA$. The atmospheric parameters were obtained using these spectra and optical observations from the literature. Element abundances were determined using theoretical models, which include the effects of element stratification due to gravitational settling and radiative levitation.

Results. We find 48 of the 85 DA white dwarfs studied, or 56\% show traces of heavy elements. In 25 stars (showing only Si and occasionally $\mathrm{C}$ ), the elements can be explained by radiative levitation alone, although we argue that accretion has very likely occurred recently. The remaining 23 white dwarfs (27\%), however, must be currently accreting. Together with previous studies from the ground and adopting bulk Earth abundances for the debris, accretion rates range from a few $10^{5} \mathrm{~g} \mathrm{~s}^{-1}$ to a few $10^{8} \mathrm{~g} \mathrm{~s}^{-1}$, with no evident trend in cooling age from $\approx 40 \mathrm{Myr}$ to $\approx 2 \mathrm{Gyr}$. Only a single, modest case of metal pollution $\left(\dot{M}<10^{6} \mathrm{~g} \mathrm{~s}^{-1}\right)$ is found among ten white dwarfs with $T_{\text {eff }}>23000 \mathrm{~K}$, in excellent agreement with the absence of infrared excess from dust around these warmer stars. The median, main sequence progenitor of our sample corresponds to an A-type star of $\approx 2 M_{\odot}$, and we find 13 of 23 white dwarfs descending from main sequence 2-3 $M_{\odot}$, late B- and A-type stars to be currently accreting. Only one of 14 targets with $M_{\text {wd }}>0.8 M_{\odot}$ is found to be currently accreting, which suggests a large fraction of these stars result from double-degenerate mergers, and the merger disks do not commonly reform large planetesimals or otherwise pollute the remnant. We reconfirm our previous finding that two $625 \mathrm{Myr}$ Hyades white dwarfs are currently accreting rocky planetary debris.

Conclusions. At least $27 \%$ of all white dwarfs with cooling ages 20-200 Myr are accreting planetary debris, but that fraction could be as high as $\approx 50 \%$. At $T_{\text {eff }}>23000 \mathrm{~K}$, the luminosity of white dwarfs is probably sufficient to vaporize circumstellar dust grains, so no stars with strong metal-pollution are found. Planetesimal disruption events should occur in this cooling age and temperature range as well, and they are likely to result in short phases of high mass-transfer rates. It appears that the formation of rocky planetary material is common around 2-3 $M_{\odot}$ late B- and A-type stars.
\end{abstract}

Key words. white dwarfs - stars: atmospheres - stars: abundances - circumstellar matter - planetary systems

\section{Introduction}

It is becoming increasingly clear that the presence of planets is more the norm than the exception. Analysis of transiting planet candidates from Kepler puts the frequency of earth-sized planets with orbital periods $<85$ days at $16.5 \pm 3.6 \%$ (Fressin et al. 2013), whereas microlensing, which is sensitive to planets on longer period orbits, suggests that the fraction of stars with cool Neptunes or super Earths is $\approx 50-60 \%$ (Cassan et al. 2012).

A fundamental question that has, until recently, received surprisingly little attention is the ultimate fate of planetary systems once their host stars evolve off the main sequence. Initially focusing on the future of the solar system (Sackmann et al. 1993; Duncan \& Lissauer 1998), a number of theoretical studies have shown that a fraction of planets can survive the red-giant stage

\footnotetext{
* Table 1 is available in electronic form at http://www. aanda.org
}

of their host stars (Villaver \& Livio 2007, 2009; Nordhaus et al. 2010; Mustill \& Villaver 2012). The ensuing long-term orbital evolution is complex and may lead to planet ejections or collisions (Debes \& Sigurdsson 2002; Veras et al. 2011; Voyatzis et al. 2013). Smaller bodies are likely to be scattered, from locations comparable to the solar system's main asteroid belt or Kuiper belt (Bonsor et al. 2011; Debes et al. 2012), which will lead to their tidal disruption if their trajectory takes them within the Roche radius of the white dwarf, $\approx 1 R_{\odot}$, (Davidsson 1999).

Accretion of debris from the disruption of planetary bodies is now the canonical explanation (Graham et al. 1990; Jura 2003) for the presence of dusty and gaseous disks discovered around 30 white dwarfs (e.g., Zuckerman \& Becklin 1987; Becklin et al. 2005; Gänsicke et al. 2006; Farihi et al. 2008; Dufour et al. 2010; Kilic et al. 2012) and the presence of photospheric metals in a large number of white dwarfs. 
The strong surface gravity of white dwarfs leads to gravitational settling: all heavy elements sink out of the atmosphere with only the lightest one floating on top (Schatzman 1947). This explains the composition of the vast majority of objects with almost pure hydrogen or helium surfaces. Metals are expected at the hottest temperatures because of radiative levitation (Michaud et al. 1979; Vennes et al. 1988; Chayer et al. 1995a,b), or at the cool end of the cooling sequence through convective mixing with deeper layers (Koester et al. 1982; Fontaine et al. 1984; Pelletier et al. 1986). Nevertheless, small traces of heavy metals are also found in the intermediate temperature range (see, e.g., Koester \& Wilken 2006, for a DA sample), where they can only be supplied by an external source through accretion. Historically, accretion from interstellar matter was considered as the most likely explanation (Fontaine \& Michaud 1979; Vauclair et al. 1979; Alcock \& Illarionov 1980; Dupuis et al. 1992). However, this scenario had several problems (e.g. Aannestad et al. 1993; Wolff et al. 2002; Friedrich et al. 2004; Farihi et al. 2010a), and it is now clear that accretion of planetary debris is the most likely explanation for the majority of, if not all, metal-polluted white dwarfs.

The direct detection of planetary material in the photosphere of a white dwarf offers the opportunity to study the chemical composition of exoplanetary systems with a scope and accuracy that will not be reached by other methods in the foreseeable future (Zuckerman et al. 2007). Because high resolution and high signal-to-noise spectra are needed, detailed studies have so far been carried out only for a handful of objects (e.g. Klein et al. 2010, 2011; Vennes et al. 2011a; Zuckerman et al. 2011; Dufour et al. 2012; Gänsicke et al. 2012; Jura et al. 2012; Xu et al. 2013a), with the common conclusion that the parent bodies of the accreted debris are rocky.

The stars in these studies form a heterogenous sample that covers a wide range in effective temperatures, major element composition $(\mathrm{H} \text { or } \mathrm{He})^{1}$, and metal settling timescales from days to millions of years. In particular for those objects with extended convection zones and long diffusion timescales (cool DA and the majority of DB white dwarfs), it is usually not possible to determine whether accretion has just started, if it is now ongoing in a quasistationary state, or if it has already ended long ago. This uncertainty impedes accurate measurements of both the accretion rates and chemical abundances of the circumstellar debris (Koester 2009). Addressing the overall picture and answering questions, such as "How many and what kinds of white dwarf progenitors harbored a planetary system, and what were their compositions? Where does the reservoir of small bodies that are scattered reside, and how does it evolve with time?", requires deep, high-resolution observations of a much larger and unbiased sample.

Ground-based observations of white dwarfs are primarily sensitive to the detection of photospheric Ca via the H\&K doublet. Zuckerman et al. (2003) carried out the first systematic study, observing with the Keck HIRES echelle spectrograph primarily cool ( $T_{\text {eff }} \lesssim 10000 \mathrm{~K}$ ) and old (cooling age $\gtrsim 1 \mathrm{Gyr}$ ) DA white dwarfs, and found traces of $\mathrm{Ca}$ in $\approx 25 \%$ of their target sample. A number of additional white dwarfs with weak $\mathrm{CaH} \& \mathrm{~K}$ lines were identified in the SPY survey (Napiwotzki et al. 2001; Koester et al. 2005; Berger et al. 2005), and Koester $\&$ Wilken (2006) used this combined sample of 38 DAZ to test the hypothesis of interstellar accretion as origin of the metals. A similar, but smaller study focusing on $30 \mathrm{DB}$ white dwarfs

\footnotetext{
1 DA and DB denote white dwarfs with hydrogen and heliumdominated atmospheres, respectively. The letter " $\mathrm{Z}$ " is added to identify the presence of photospheric metals.
}

reached comparable conclusions, $\approx 25 \%$ of these stars have remnants of planetary systems (Zuckerman et al. 2010), with the statistics subject to the caveat regarding stars with deep convection zones mentioned above. Fundamental limitation of all these ground-based studies are that (1) for a given photospheric $\mathrm{Ca}$ abundance, the strength of the $\mathrm{H} \& \mathrm{~K}$ doublet varies by many orders of magnitude for white dwarf temperatures ranging from $10000 \mathrm{~K}$ to $25000 \mathrm{~K}$, with corresponding cooling ages of a few 10 Myr to nearly $1 \mathrm{Gyr}$, and (2) Ca is only a modest component of rocky planets, $<2 \%$ of the bulk Earth (McDonough 2000).

Space-based observations of white dwarfs are sensitive to many additional polluting elements, but have so far focused predominantly on hot $(>30000 \mathrm{~K})$ white dwarfs. While a substantial number of hot white dwarfs showing photospheric metals have been detected (Shipman et al. 1995; Bannister et al. 2003; Barstow et al. 2003; Dickinson et al. 2012a,b), the interpretation of their origin has been complicated by the strong effects of radiative levitation in their atmospheres, though Barstow et al. (2014) argue for external pollution.

Here, we present the results of a well defined large farultraviolet spectroscopic survey of warm (17 000-27000 K) DA white dwarfs that is sensitive to many of the major components of rocky material $(\mathrm{O}, \mathrm{Fe}$, and in particular $\mathrm{Si})$ and extends our knowledge regarding the frequency of planetary debris around white dwarfs and their main sequence progenitors to younger cooling ages $(\approx 20-200 \mathrm{Myr})$. In this temperature range, DA white dwarfs have very simple atmospheres, no convection zones, and short diffusion timescales. It is therefore safe to assume that the diffusion occurs in a steady state and the calculation of the chemical composition of the accreted matter is straightforward. One complication in this range - radiative levitation of silicon and carbon - was not anticipated at the beginning of the project, but we developed the necessary calculations to account for this effect.

\section{Sample selection and HST observations}

We selected an input target list of 150 DA white dwarfs from Liebert et al. (2005) and Koester et al. (2009), where the sole criteria were that the stars (a) had $17000 \mathrm{~K}<T_{\text {eff }}<25000 \mathrm{~K}$ (two targets turned out to be slightly hotter in our analysis, extending the temperature range to $\approx 27000 \mathrm{~K}$ ), and (b) had predicted fluxes $F_{\lambda}(1300 \AA)>5 \times 10^{-14} \mathrm{erg} \mathrm{cm}^{-2} \mathrm{~s}^{-1} \AA^{-1}$. Given the need to obtain far-ultraviolet spectroscopy for a large sample of DA white dwarfs, but with no specific need to observe any particular star, this project was implemented as an HST Snapshot Program. Such snapshots are short observations occupying at most one HST orbit, which are executed in gaps of the scheduled observing sequence, where no other target from the pool of accepted regular GO or Large programs is available. We included several types of ancillary targets to facilitate the comparison with the main DA sample, and to test our computational tools: several white dwarfs with close M-dwarf companions (drawn from Schreiber \& Gänsicke 2003 and Farihi et al. 2010b), two recently discovered metal-polluted DA white dwarfs from Vennes et al. (2010, 2011b), and a small number of bright DB white dwarfs from Bergeron et al. (2011). This HST program was executed from September 2010 to February 2013, and a total of 85 DA white dwarfs from our target list, plus 15 ancillary targets were successfully observed. The distribution of the fundamental parameters of the $85 \mathrm{DA}$ white dwarfs $\left(T_{\mathrm{eff}}, \log g, V\right.$-band magnitude, $M_{\mathrm{wd}}$, cooling age, and progenitor mass $M_{\mathrm{ms}}$ ) are illustrated in Fig. 1. 
D. Koester et al.: The frequency of planetary debris around young white dwarfs

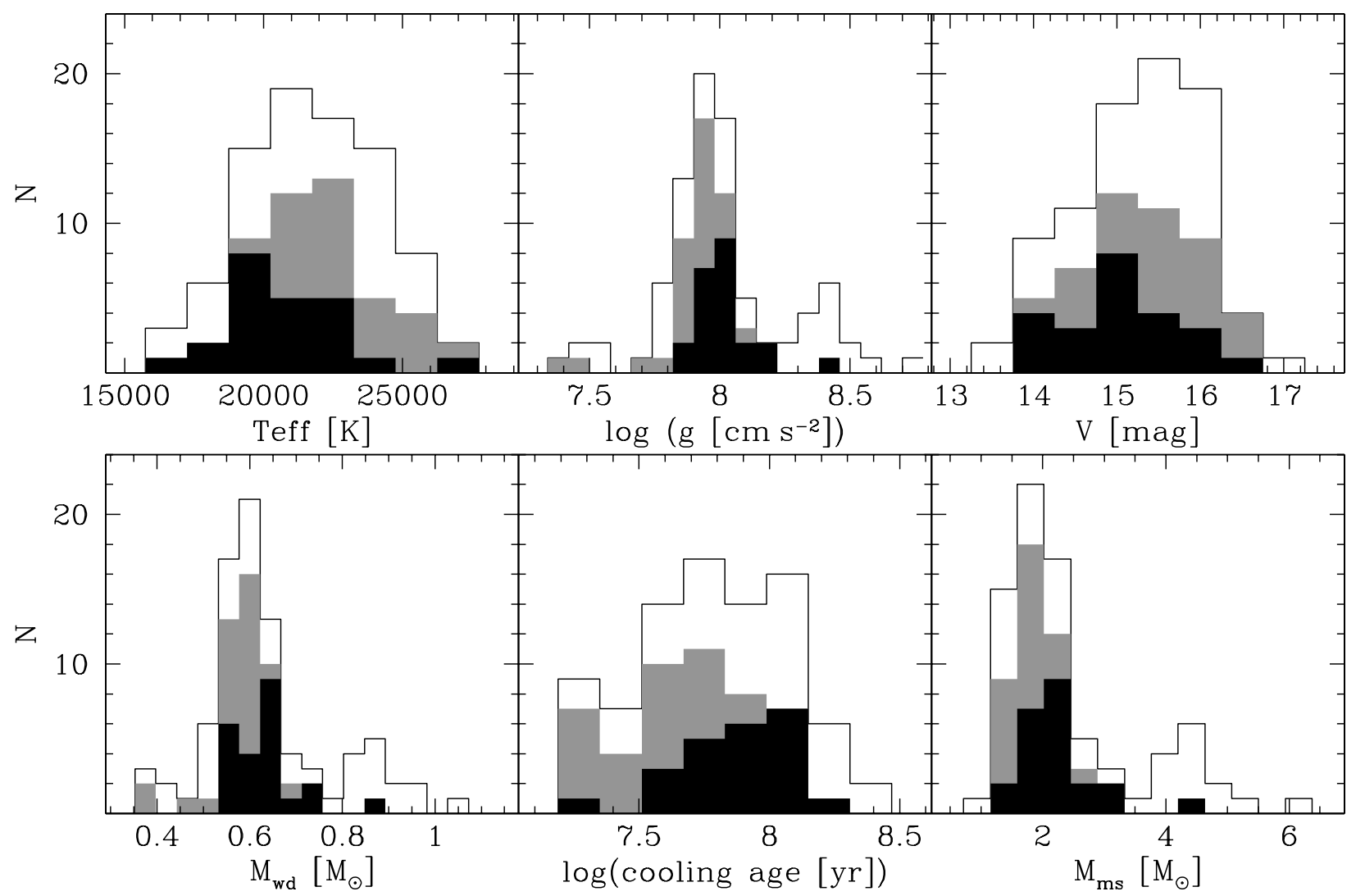

Fig. 1. Fundamental properties of the DA stars observed in this HST/COS far-ultraviolet spectroscopic survey. From top left to bottom right: effective temperature and surface gravity (Sect. 3), $V$-band magnitude, white dwarf mass and cooling age computed from $T_{\text {eff }}$ and log $g$ using the cooling models of Fontaine et al. (2001), and the main-sequence progenitor mass computed from $M_{\mathrm{wd}}$ and the initial-to-final mass relation of Kalirai et al. (2008). The full sample is indicated by the outlined histogram. 48 white dwarfs where at least photospheric Si has been detected are shown with the filled histograms, of which 23 must be currently accreting (black). The remaining 25 (grey) have Si abundances consistent with support from radiative levitation alone, and have very likely accreted in the recent past (Sect. 9.1).

All observations were obtained with the Cosmic Origins Spectrograph (COS, Green et al. 2012) using the G130M grating. We adopted a central wavelength of $1291 \AA$, which covers the wavelength range from 1130 to $1435 \AA$, with a gap at 1278-1288 $\AA$ due to the space between the two detector segments. The COS detector suffers of a number of fixed-pattern problems, which can be eliminated by obtaining multiple spectra dithered by small steps in the dispersion direction, so-called FP-POS settings. The best results are obtained using all available four FP-POS settings, but given the limited time available in a snapshot exposure, we chose to split the exposure time equally between only two FP-POS positions (1 and 4).

Several of our COS observations were already used for the study of individual objects, including a detailed abundance study of four strongly metal-polluted DAZ stars that also exhibit infrared excess from circumstellar dust (Gänsicke et al. 2012); the detection of planetary debris at two white dwarfs in the Hyades (Farihi et al. 2013b, see also Sect. 9.6) and of water in an extrasolar minor planet (Farihi et al. 2013a); and the identification of molecular hydrogen in one DA white dwarf that turned out to be significantly cooler than the published effective temperatures (Xu et al. 2013b; Zuckerman et al. 2013).

In this paper, we focus on the statistical analysis of the DA sample, a more detailed discussion of the white dwarfs that exhibit photospheric absorption in addition to $\mathrm{Si}$ and $\mathrm{C}$ will be discussed elsewhere.

\section{Atmospheric parameters}

The parameters were determined by comparison of the observed spectra with a grid of theoretical models, calculated with the methods and input physics as described in Koester (2010). The only important change compared to the grid used for the analysis of the SPY survey (Koester et al. 2009) are the inclusion of the non-ideal Balmer line Stark profiles as calculated by Tremblay $\&$ Bergeron (2009) and new Lyman $\alpha$ profiles.

The new calculations for the broadening of the Lyman $\alpha$ line, and in particular the quasi-molecular satellites at 1400 and $1600 \AA$, use the unified theory as described in Allard \& Kielkopf (1982) and Allard et al. (1999). The program code for the profiles, however, has been completely rewritten with improved numerical algorithms to overcome problems with numerical noise and artifacts, caused by the very large dynamical range of ten or more orders of magnitude between the line core absorption and the far wing in very broad lines. We have also used new calculations for the adiabatic potentials and dipole moments for the $\mathrm{H}-\mathrm{H}+$ interaction by Santos \& Kepler (2012).

The HST/COS spectra are dominated by the Lyman $\alpha$ profile, including the satellite near $1400 \AA$, and contain practically no undisturbed continuum. Although a fit can be obtained with effective temperature $T_{\text {eff }}$ and surface gravity $\log g$ as free parameters, the result is quite uncertain. A small increase in $\log g$ can almost perfectly be compensated by an increase in $T_{\text {eff }}$, such that the ionization fraction, the major determinant for 
atmospheric structure and line strengths, remains constant. Very minor changes in the fitting routine cause the solution to wander along this correlation line. It is therefore necessary to use additional information to fix either the temperature or $\log g$ in the fitting of the COS spectra. Fortunately all objects have recent parameter determinations from high quality optical spectra, which do not suffer from this degeneracy, and up-to-date model atmospheres.

Seventy one of the 90 objects (including five of the ancillary targets) have been analyzed from optical spectra by Gianninas et al. (2011, hereafter G11), and 60 objects have at least two spectra in the SPY database. The latter have been reanalyzed by us using the latest model grid. The overlap of both samples (excluding WD 0933+025 and WD 1049+103 with strong contribution from a red companion in the optical [G11]) contains 39 objects, which can be used to estimate systematic and statistical errors of the parameter determination.

The G11 temperatures are on average higher by $3.16 \%$, the surface gravities are larger by 0.056 dex. One possible explanation for these differences could be the theoretical models, but we believe that we use essentially the same input physics as the Montreal group. The models are rather simple with no convection, fairly low matter densities, and weak non-ideal effects. When we implemented the Tremblay \& Bergeron (2009) Stark broadening data, P. Tremblay (2009, priv. comm.) provided a theoretical spectrum for the optical range of a DA at $18000 \mathrm{~K}$, $\log g=8$. The corresponding model in our current grid is within $<1 \%$ of the flux identical to that benchmark spectrum. We thus do not believe that the different results come from differences in the theoretical models. The different nature of the observations (e.g. echelle vs. long slit spectra), or different fitting methods remain as possible explanations.

Apart from the systematic differences there is also a statistical scatter, which we interpret as an indication of the statistical error in our parameter determination $\left(\sigma\left(T_{\text {eff }}\right) \approx 2.36 \%\right.$, $\sigma(\log g) \approx 0.084)$. Figure 2 shows the comparison for the common sample.

The systematic difference in the optical parameter determination between G11 and the current work leads to different predictions for the theoretical UV spectra. The $T_{\text {eff }}$ difference has a much larger effect than that of $\log g$; we have therefore decided to adopt the surface gravities from optical results and use $T_{\text {eff }}$ as free fit parameter for the COS spectra. Keeping our own optical surface gravities fixed we obtain temperatures from the ultraviolet fitting, which are similar to our optical temperatures, with an average difference of only $80 \mathrm{~K}$. On the other hand, using the G11 surface gravities, our fits to the COS spectra results in temperatures, which are on average $600 \mathrm{~K}$ lower than found by G11 from their optical spectra. In simple words: using our own models and the described method, the parameters are consistent between optical and ultraviolet fitting, whereas for the G11 parameters they are not. We do not know if a fit of the ultraviolet spectra with the Montreal models would give more consistent results, but the implication would be that their theoretical Lyman $\alpha$ spectra are different from our current implementation.

The derived (COS) temperatures using either the G11 or our $\log g$ values from the SPY spectra differ by only $169 \mathrm{~K}$ on average, much less than they differ from the G11 temperatures, as shown above, with a distribution completely determined by the statistical errors of the optical surface gravities. The statistical (formal) errors for $T_{\text {eff }}$ from the fit routines are small (about $20 \mathrm{~K}$ typically), and can be neglected entirely. This results in a tight correlation between $\log g$ and the resulting COS temperature, which we use in the error estimates below.
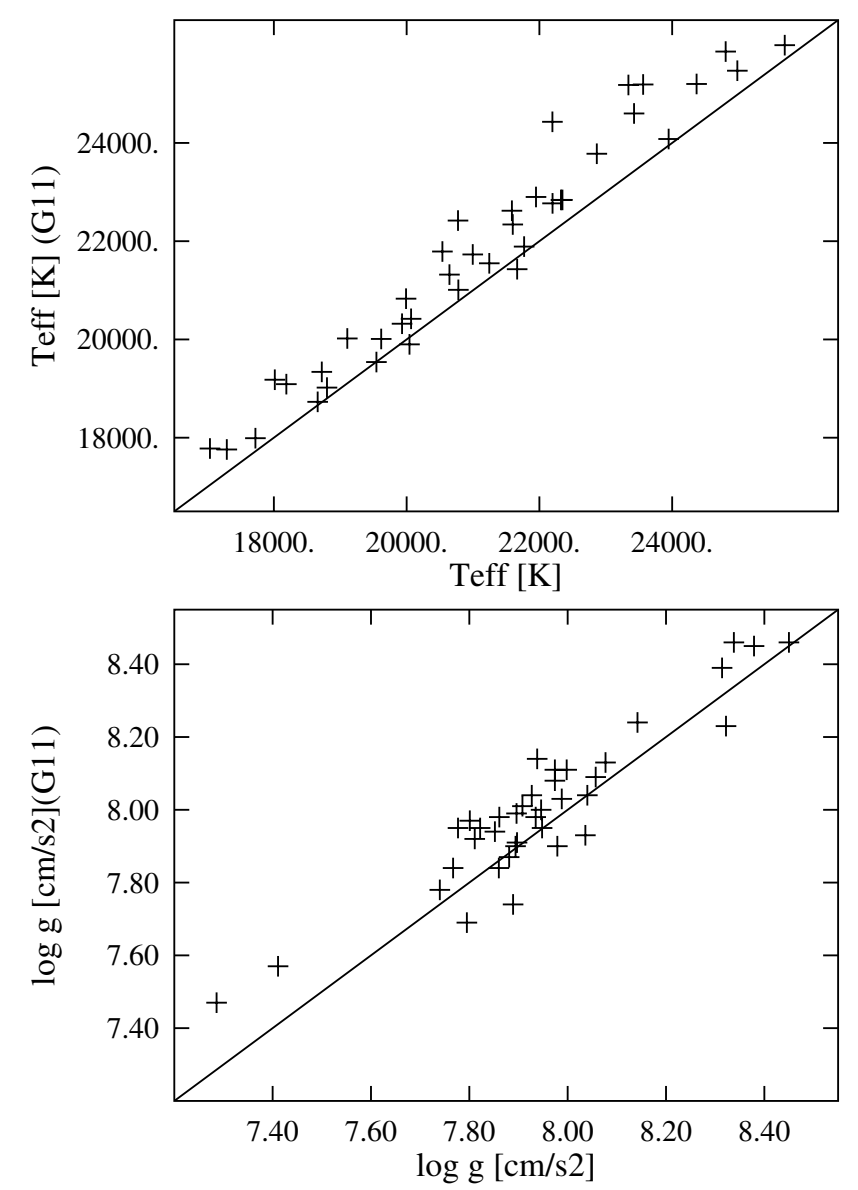

Fig. 2. Comparison of $T_{\text {eff }}$ and $\log g$ between the Gianninas et al. (2011) results and our own determinations from the reanalysis of the SPY spectra. The continuous lines are the 1:1 relations.

These experiments suggested the following procedure. For the overlapping sample between G11 and SPY we take the average $\log g$. For those with only G11 determination we decrease $\log g$ by one half the systematic difference (0.028), whereas for those with SPY determination alone, we increase $\log g$ by the same amount. This decreases the systematic difference to either sample to \pm 0.028 in $\log g$; adding the statistical error quadratically results in $\sigma(\log g)=0.089$. With this $\log g$ kept fixed, we fit the ultraviolet spectra to determine a (COS) temperature. The error of $\log g$ translates into an error of $T_{\text {eff }}$ of $1.36 \%$. We therefore use as our final error estimates $\sigma\left(T_{\text {eff }}\right)=$ $1.36 \%$ and $\sigma(\log g)=0.089$, with a very tight correlation (larger $\log g$ leads to larger $T_{\text {eff }}$ ). The results are presented in Table 1, which also contains photometric $V$ data from the SIMBAD database, the Villanova White Dwarf Catalog ${ }^{2}$, and the APASS Photometric All-Sky Survey ${ }^{3}$, and some derived quantities. Masses and radii are obtained from the Montreal evolutionary calculations ${ }^{4}$ (Fontaine et al. 2001), two independent distance estimates from the comparison of the absolute HST flux and the $V$ magnitude with theoretical predictions from the model atmosphere parameters and the radius. A comparison of both values is shown in Fig. 3.

\footnotetext{
2 http://www.astronomy.villanova.edu/WDcatalog/

3 http://www. aavso.org/apass

4 http://www. astro. umontreal.ca/ bergeron/

CoolingModels/
} 
D. Koester et al.: The frequency of planetary debris around young white dwarfs

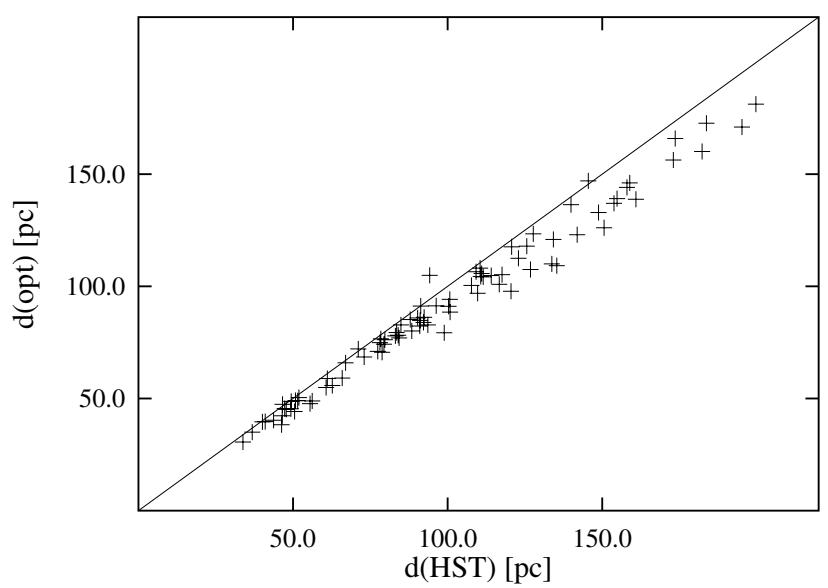

Fig. 3. Comparison of distances derived using the $V$ magnitude vs. using the absolute calibration of COS. The systematic deviation from the 1:1 relation (solid line) is caused by our neglect of interstellar reddening with a much stronger effect in the ultraviolet than on $V$.

The systematic difference between the two values indicates that some amount of reddening is present for the more distant objects. Assuming a $1 / \lambda$ dependence leads to an $\approx 20 \%$ change of the absorption over the broad $\mathrm{L} \alpha$ line. In our spectral fitting we use the fixed surface gravity from the optical determination. The temperature is then obtained from a fit to the $\mathrm{L} \alpha$ line, which is dominated by the overall strength (i.e. the equivalent width) of this line, unaffected by the reddening.

\section{Element abundances}

Atmospheric structures were calculated for the best fit parameters with the same input physics used in constructing the grid. With this model structure synthetic spectra were calculated including approximately 14000 spectral lines of 14 elements. The elements were assumed to be homogeneously distributed throughout the atmosphere. Individual abundances were then varied until a satisfactory fit to the spectral lines in the observed spectrum was achieved. We then calculated models with the metal abundances changed by \pm 0.2 dex and estimated the abundance errors from these models. Table 2 shows the results for the $\mathrm{Si}$ and $\mathrm{C}$ abundances. Figure 4 shows an example for the total range of the observed spectra, as well as details of the spectral ranges with the $\mathrm{Si}$ and $\mathrm{C}$ lines important for the present study, compared with model fits. The panels from top to bottom show:

a) Complete COS spectral range for WD 1943+163 with typical signal-to-noise and relatively large $\mathrm{Si}$ and $\mathrm{C}$ abundances.

b) Si II 1260.422, 1264.738, 1265.002 $\AA$. Note the strong blue-shifted interstellar component of the resonance line $1260.422 \AA$. The sulfur S I $1259.519 \AA$ line is also visible at the photospheric position.

c) Si III 1294.545, 1296.726, 1298.892, 1298.946, $1301.149 \AA$ lines.

d) Carbon, C II 1334.530, 1335.660, $1335.708 \AA$ lines, with interstellar component of the resonance line $1334.530 \AA$.

e) C III 1174.930, 1175.260, 1175.590, 1175.710, 1175.987, $1176.370 \AA$ lines. These C III lines are weak in WD 1943+163; the spectrum shown is for HE 0416-1034. The continuous (red) lines are the theoretical model.

Somewhat surprisingly, of the 85 sample objects in Table 2, 48 show photospheric $\mathrm{Si}$. In fact, $\mathrm{Si}$ is always present, if any heavy
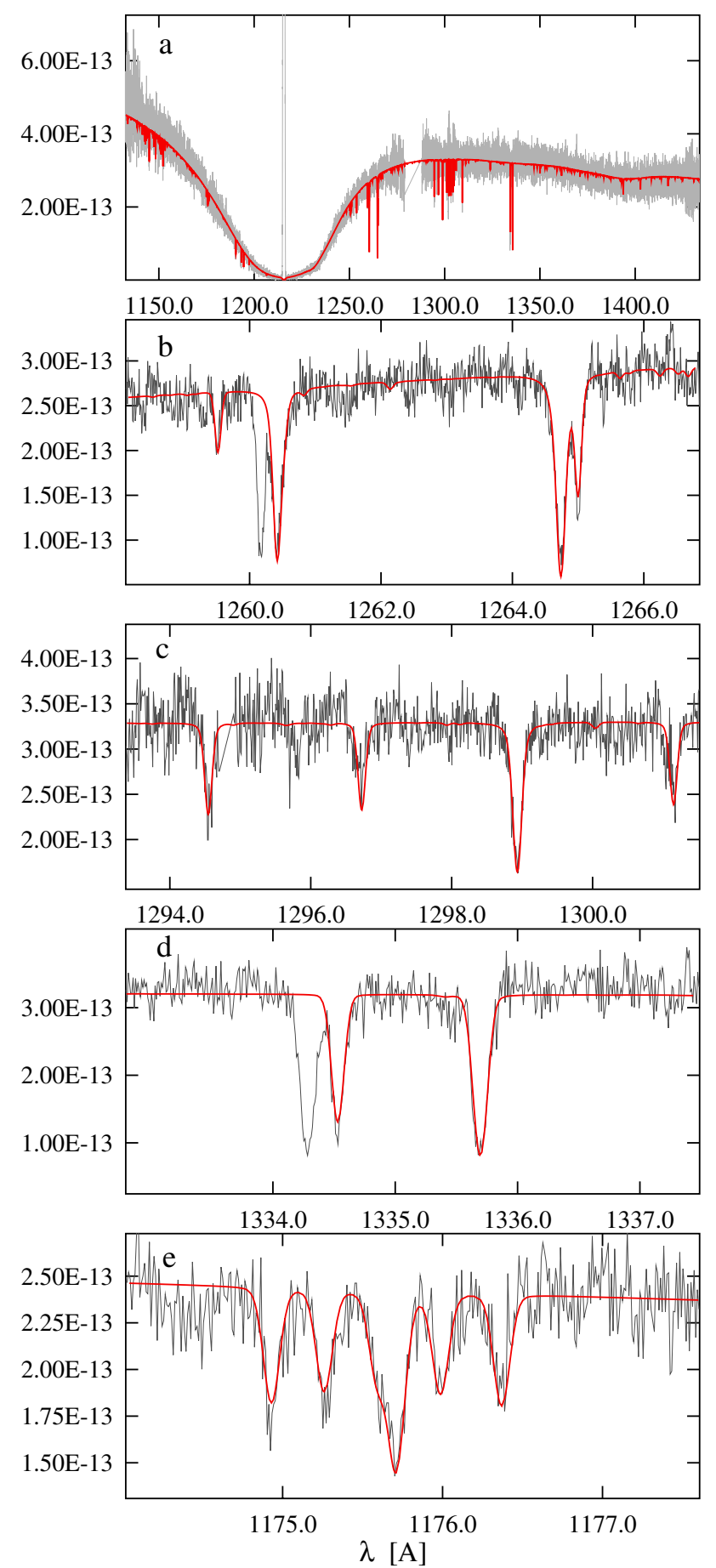

Fig. 4. Example spectra with the important lines for this work. The vertical axis is the observed flux in erg $\mathrm{cm}^{-2} \mathrm{~s}^{-1} \AA^{-1}$.

metals are seen. 18 of these show in addition $\mathrm{C}$, and 7 further metals out of this list: $\mathrm{Mg}, \mathrm{Al}, \mathrm{P}, \mathrm{S}, \mathrm{Ca}, \mathrm{Cr}, \mathrm{Fe}, \mathrm{Ni}$. For reasons, which will become clear in the following, we will concentrate in this paper on the discussion of the $\mathrm{Si}$ and $\mathrm{C}$ abundances. The very interesting cases with more heavy metals than these two will be treated in separate papers.

\subsection{Effect of parameter errors on the abundances}

As a test case we have used WD 1943+163, near the middle of the temperature range and with $\mathrm{Si}, \mathrm{C}$, and $\mathrm{O}$ detected. 
Table 2. Si and C abundances.

\begin{tabular}{|c|c|c|c|c|c|c|c|}
\hline Object & $\log [\mathrm{Si} / \mathrm{H}]$ & $\log [\mathrm{C} / \mathrm{H}]$ & Notes & Object & $\log [\mathrm{Si} / \mathrm{H}]$ & $\log [\mathrm{C} / \mathrm{H}]$ & Notes \\
\hline WD $0000+171$ & $-8.10(0.15)$ & -8.30 & & WD $1102+748$ & -8.50 & & \\
\hline WD 0013-241 & -8.40 & & & WD 1104+602 & -8.40 & & \\
\hline WD 0018-339 & -8.00 & & & WD $1129+155$ & $-8.30(0.25)$ & -8.10 & \\
\hline WD 0028-474 & -8.40 & & & WD $1133+293$ & $-7.70(0.15)$ & -7.70 & \\
\hline WD 0047-524 & -8.40 & & & SDSS $1228+1040$ & $-5.20(0.10)$ & $-7.60(0.20)$ & $*, 1,4,5$ \\
\hline WD 0059+257 & $-6.50(0.10)$ & -8.00 & 1 & WD 1229-013 & -8.30 & & \\
\hline WD 0102+095 & $-7.35(0.15)$ & $-8.00(0.20)$ & & WD 1230-308 & -8.10 & & \\
\hline WD 0114-605 & $-7.40(0.20)$ & $-8.10(0.20)$ & & HS1243+0132 & $-7.80(0.15)$ & -8.30 & \\
\hline WD 0124-257 & -8.20 & & & WD $1257+048$ & $-7.50(0.15)$ & $-7.70(0.20)$ & \\
\hline WD 0140-392 & $-7.50(0.15)$ & -8.40 & & WD 1310-305 & $-7.70(0.15)$ & -8.10 & \\
\hline WD 0155+069 & -8.30 & & & WD $1325+279$ & $-8.00(0.15)$ & -8.20 & \\
\hline HS0200+2449 & -8.30 & & & WD 1325-089 & $-8.60(0.25)$ & -8.00 & \\
\hline WD 0242-174 & $-8.20(0.20)$ & -8.30 & & WD $1330+473$ & -8.30 & & \\
\hline WD $0307+149$ & $-7.85(0.15)$ & -8.30 & & WD $1353+409$ & -7.80 & & \\
\hline WD $0308+188$ & -8.20 & & & WD $1408+323$ & $-7.70(0.15)$ & $-8.30(0.20)$ & \\
\hline HE0308-2305 & -7.80 & & & WD $1451+006$ & $-7.70(0.20)$ & -8.20 & \\
\hline WD $0341+021$ & $-7.95(0.20)$ & $-7.55(0.15)$ & & WD $1459+347$ & -8.20 & & \\
\hline WD $0352+018$ & $-8.10(0.15)$ & -8.30 & & WD 1524-749 & -7.90 & & \\
\hline HE0358-5127 & -7.80 & & & WD 1531-022 & -8.40 & & \\
\hline HE0403-4129 & $-7.10(0.15)$ & -7.90 & & WD $1547+057$ & -8.20 & & \\
\hline HE0414-4039 & -8.20 & & & WD $1548+149$ & $-7.65(0.15)$ & $-7.40(0.15)$ & \\
\hline HE0416-1034 & $-7.35(0.15)$ & $-7.05(0.20)$ & & WD $1633+676$ & -7.90 & & \\
\hline HE0418-1021 & -8.20 & & & WD $1647+375$ & $-6.20(0.10)$ & $-5.50(0.20)$ & 1 \\
\hline WD $0421+162$ & $-7.50(0.15)$ & -8.30 & & WD $1713+332$ & $-8.30(0.25)$ & $-7.60(0.20)$ & \\
\hline WD $0431+126$ & $-8.10(0.15)$ & -8.30 & & WD $1755+194$ & $-7.30(0.20)$ & $-8.10(0.20)$ & \\
\hline HE0452-3444 & $-7.60(0.15)$ & -8.20 & & WD 1914-598 & $-8.30(0.15)$ & -8.30 & \\
\hline HS0507+0434A & $-8.40(0.15)$ & -8.50 & & WD 1929+012 & $-4.75(0.15)$ & $-6.80(0.30)$ & $*, 1,4$ \\
\hline WD $0710+741$ & $-6.70(0.15)$ & $-5.90(0.30)$ & $*, 1,2$ & WD $1943+163$ & $-6.80(0.10)$ & $-6.60(0.20)$ & 1 \\
\hline WD $0843+516$ & $-5.20(0.10)$ & $-7.20(0.20)$ & 1,4 & WD 1953-715 & $-6.70(0.10)$ & $-6.60(0.20)$ & 1 \\
\hline WD $0854+404$ & $-7.90(0.20)$ & -8.10 & & WD 2021-128 & -8.30 & & \\
\hline WD $0920+363$ & $-7.30(0.20)$ & -7.70 & & WD $2032+188$ & -8.20 & & \\
\hline WD $0933+025$ & -8.30 & & 3 & WD 2046-220 & $-7.60(0.15)$ & -7.60 & \\
\hline HS0944+1913 & -8.40 & & & WD $2058+181$ & $-7.50(0.10)$ & $-6.50(0.20)$ & 1 \\
\hline WD $0947+325$ & -8.30 & & & WD $2134+218$ & $-8.10(0.15)$ & -8.10 & \\
\hline WD 0954+697 & $-8.00(0.20)$ & -8.20 & & HS2210+2323 & -8.20 & & \\
\hline WD $1005+642$ & -8.20 & & & WD $2220+133$ & -8.20 & & \\
\hline WD $1013+256$ & $-6.90(0.10)$ & $-6.40(0.20)$ & & HS2229+2335 & $-6.50(0.15)$ & $-6.70(0.20)$ & \\
\hline WD $1015+161$ & $-6.40(0.10)$ & -8.00 & 1,4 & HE2231-2647 & $-8.10(0.15)$ & -8.40 & \\
\hline WD $1017+125$ & $-7.50(0.15)$ & -7.60 & & HE2238-0433 & -7.50 & & \\
\hline WD 1034+492 & $-7.55(0.10)$ & -8.00 & & HS2244+2103 & $-7.70(0.15)$ & -8.10 & \\
\hline WD $1038+633$ & $-7.90(0.30)$ & -8.00 & & WD $2256+249$ & $-6.85(0.10)$ & $-6.10(0.20)$ & $*, 1,2$ \\
\hline WD $1049+103$ & -8.40 & & 3 & WD $2257+162$ & $-7.40(0.15)$ & $-6.40(0.20)$ & $*, 2$ \\
\hline WD 1049-158 & -8.40 & & & WD $2306+124$ & $-7.60(0.10)$ & $-8.00(0.20)$ & \\
\hline WD $1052+273$ & -8.50 & & & WD 2322-181 & $-7.70(0.10)$ & $-7.70(0.15)$ & \\
\hline WD 1058-129 & -8.30 & & & WD 2359-324 & -7.80 & & \\
\hline
\end{tabular}

Notes. Abundances are logarithm of the metal to hydrogen ratio by numbers. Numbers in parenthesis are estimated errors. If absent, the abundances are upper limits (Sect. 9). *: Ancillary target, not used in sample statistics; 1: additional elements detected among the following: O, Mg, Al, P, S, $\mathrm{Ca}, \mathrm{Fe}$ in the ultraviolet or optical spectra; 2: post-common envelope (close) binary; 3 : spatially resolved (wide) binary; 4: exhibits excess infrared emission; 5: SDSS1228 is a gas-disk DAZ with emission lines seen in the infrared Ca II triplet near $8500 \AA$ (Gänsicke et al. 2006) and the Mg II doublet at $\approx 2800 \AA$ (Hartmann et al. 2011). The disk emission lines are broad - in contrast, the photospheric absorption lines are narrow, and we would expect to detect any emission in Si in the high-quality COS spectra of SDSS 1228+1040. In addition, the computations of Hartmann et al. (2011) predict little emission in Si II, and none in Si III. We use many Si II and Si III in the abundance analysis and do not see a significant difference.

Calculating two new models with the parameters increased, respectively decreased by the above uncertainties, the changes in the derived abundances are smaller than 0.04 dex for all lines, which we take as an estimate for the uncertainty caused by parameter errors, which should be added quadratically to the errors in Table 2.

\section{Interstellar absorption lines}

With the exception of SDSS 1228+1040 and HS 2229+2335, where the photospheric Si lines are exceptionally strong and probably mask the IS lines, all objects show interstellar lines of Si II $1260 \AA$ (and others) and C II 1334/35. . In most objects 
lines from the ground state of $\mathrm{O}, \mathrm{N}, \mathrm{S}, \mathrm{Fe}$ are also visible. The most obvious indication for their IS nature is the absence of Si II $1265 \AA$, which is always stronger than the $1260 \AA$ line in the photosphere, because of the $g f$ ratio of $\approx 1.8$ and level energy only $0.036 \mathrm{eV}$ above the ground state. If Si II $1265 \AA$ is visible, it is always photospheric only and in almost all cases the interstellar component of Si II $1260 \AA$ is shifted by 0.1 to $0.3 \AA$. In addition several stars show additional Si lines from excited states, which must have a photospheric origin.

The situation is more complicated for the C II 1334/35 doublet, where the second level is only $0.008 \mathrm{eV}$ above zero, and both components can be observed in the ISM. Determination of photospheric abundances is only possible when photospheric and ISM lines are clearly separated, or if lines from excited states are observed, such as the C III $1175 \AA$ multiplet.

The spectral resolution of $\approx 17 \mathrm{~km} \mathrm{~s}^{-1}$ does not allow individual components in the IS lines to be identified. However, the equivalent widths of the IS Si II $1260 \AA$ lines in those objects without photospheric metals range from 21 to $203 \mathrm{~m} \AA$, corresponding to column densities of $10^{12}$ to $10^{13} \mathrm{~cm}^{-2}$, if interpreted as single line on the linear part of the curve of growth (e.g. Savage \& Sembach 1996). These column densities are typical values for the local interstellar cloud (LIC) surrounding our solar system (Redfield \& Linsky 2004); given the proximity of our targets, it is likely the LIC or at most one or two similar small clouds are the only absorbing ISM material between us and most of our sample. The IS lines in these 90 objects close to the sun constitute an important resource for the study of the ISM in our immediate neighbourhood and will be analyzed in another paper.

\section{Interpretation of abundances in the presence of diffusion}

It is well known that the abundances in the photosphere cannot directly be taken to infer the abundances in the accreted matter. They are modified by the diffusion processes (Koester 2009). In the temperature range of our sample $(17000-27000 \mathrm{~K})$ there is no convection zone in a DA, and thus no homogeneous reservoir, which can be used to define a diffusion timescale at the bottom of this zone. The diffusion timescale is ill-defined in this case and depends on the layer in the atmosphere taken as the reference most reasonably at the Rosseland optical depth $2 / 3$. However defined in detail, they are extremely short, of the order of days or a few years at most. It is therefore reasonable to assume a steady state between accretion and diffusion, which eliminates some of the uncertainties plaguing such studies for cooler DA or DB stars with deep convection zones. Steady state means a constant diffusion flow of each element throughout the atmosphere, which is equal to the accretion flow

$X \rho v_{\text {diff }}=$ const. $=X_{\text {acc }} \dot{m}_{\text {acc }}$.

Here, $X$ is the mass fraction of the element in question, $\rho$ the mass density in the atmosphere, and $v_{\text {diff }}$ the diffusion velocity. Since the structure of the atmosphere model is known, the depth dependence of $\rho$ and $v_{\text {diff }}$ can be calculated and leads to a predicted stratified element abundance $X(r) . X_{\text {acc }}$ is the abundance in the accreted matter and $\dot{m}_{\text {acc }}$ the accretion flow in units of $\mathrm{g} \mathrm{cm}^{-2} \mathrm{~s}^{-1}$. This shows that the abundance ratios in the accreted matter can be calculated once the diffusion fluxes for the elements are known. The diffusion fluxes are thus the final parameters in the abundance analysis, replacing the usual photospheric values. As a consequence, in the next step we have calculated synthetic spectra with stratified abundances, and adjusted these fluxes until the spectra are fit satisfactorily. When using the well known terms of gravitation, electric field, and concentration gradient in the diffusion equation, the abundances near optical depth $2 / 3$ do not change very much (typically $<0.1 \mathrm{dex}$ ) and the influence on the derived accretion abundances is noticeable, but minor. This changes drastically, however, if radiative levitation of some elements becomes important.

\section{Diffusion and radiative levitation}

Radiative levitation, the selective acceleration of individual ions by transfer of momentum from photons, has been studied extensively in the past (e.g. Chayer et al. 1995a,b, and references therein), but was usually considered important only at temperatures above 30000 K. However, Chayer \& Dupuis (2010) have demonstrated that radiative levitation could support $\mathrm{Si}$ at a low level in a DA atmosphere model with $T_{\text {eff }}=20000 \mathrm{~K}$ and $\log g=8$. Marginal support could also be possible for C. An application of this result to four DAZ white dwarfs in the temperature range 18000-25000 K (Dupuis et al. 2010) showed that in three cases the Si abundance could be explained without current accretion. Very recently, Chayer (2014) studied two DA stars in the Hyades (WD 0421+162 and WD 0431+126), which are also in our sample, and demonstrated that, according to their calculations, the Si in WD 0431+126 could be completely supported by radiative levitation (see however Sect. 9.6, where our findings differ).

This raises the possibility that the numerous objects with $\mathrm{Si}$ detections in our sample may not currently undergo an accretion episode, or that at least the derived accretion rate could be significantly altered by radiative levitation.

We have therefore decided to include this effect in our analysis, which aims to determine accretion rates from observed photospheric abundances. We start with the description of diffusion following the basic equations in Gonzalez et al. (1995) and Vennes et al. (1988), with some changes in notation and simplified for a trace element 2 in background element 1 (i.e. abundance ratio $\gamma=0$ )

$$
\begin{aligned}
v_{i}= & D_{i}\left[-\frac{\partial \ln c_{2}}{\partial r}+\left(\frac{Z_{i}}{Z_{1}} A_{1}-A_{2}\right) \frac{m g}{k T}\right. \\
& \left.+\left(\frac{Z_{i}}{Z_{1}}-1\right) \frac{\partial \ln p_{1}}{\partial r}+\frac{A_{2} m g_{\mathrm{rad}, i}}{k T}\right] .
\end{aligned}
$$

Here $A_{1}, A_{2}$ are the atomic mass numbers of the two elements, $Z_{1}$ is the average charge of the background, $Z_{i}$ the charge of ion $i$ of the trace element, $c_{2}$ its number fraction, $m$ the atomic mass unit, $k$ the Boltzmann constant, $T$ temperature, $g$ the gravitational acceleration, and $p_{1}$ the partial pressure of the ions of element 1 . $D_{i}$ and $v_{i}$ are the diffusion constants and diffusion velocity for ion $i$ of the trace element.

The radiative force on ion $i$ of the trace element is

$A_{2} m g_{\mathrm{rad}, i}=\frac{1}{c} \int_{0}^{\infty} \sigma_{i} F_{\lambda} \mathrm{d} \lambda=f_{\mathrm{rad}, i}$

where $F_{\lambda}$ is the radiative energy flux in the atmosphere, $\sigma_{i}$ the line absorption cross section of ion $i$, and $c$ the velocity of light.

There has been some discussion in the literature, starting with Gonzalez et al. (1995), about the distribution of the momentum gained through photon absorption on the different ionization stages (e.g. Chayer et al. 1995a,b). The question is, whether the absorbing ion ionizes to the next higher state before losing 
the gained momentum or not. Since the detailed calculations for each transition are much too complicated to be incorporated into our present analysis, we follow the simple prescription of Chayer et al. (1995b): if the principal quantum number of the final state of the transition is not higher than that of the ground state plus one, the momentum is lost in the absorbing ion, otherwise in the next higher state. This is implemented in the following way: dividing the radiative force in the two contributions according to the above criterion (index 1 for the momentum lost by the same ion that has absorbed it, index 2 for the momentum lost in the next higher ionization state)

$f_{\mathrm{rad}, i}=f_{1, \mathrm{rad}, i}+f_{2, \mathrm{rad}, i-1}$

we can with the number density $n$ write the total force on a unit volume as

$$
\begin{aligned}
n f_{\mathrm{rad}} & =\sum_{i} n_{i}\left(f_{1, \mathrm{rad}, i}+f_{2, \mathrm{rad}, i-1}\right) \\
& =n_{1} f_{1, \mathrm{rad}, 1}+\sum_{i=2} n_{i}\left(f_{1, \mathrm{rad}, i}+\frac{n_{i-1}}{n_{i}} f_{2, \mathrm{rad}, i-1}\right) .
\end{aligned}
$$

This outlines how the effective radiative acceleration for each ion should be calculated to preserve the total absorbed momentum. The final step is the averaging of the velocities, weighting with the ionization fractions of the trace element,

$v=\sum_{i} \frac{n_{i}}{n} v_{i}$

and of the diffusion flux in $\mathrm{g} \mathrm{cm}^{-2} \mathrm{~s}^{-1}$

$f_{\text {diff }}=X \rho v$

with mass density $\rho$ and mass fraction $X$ of the trace element.

We note that all necessary quantities are readily available in the atmosphere model code during the iteration of the atmospheric structure; there is no need for any further approximations of the radiative flux or line profiles. The atmospheric structure and element distribution are iterated until a completely consistent solution is obtained for the parameters $T_{\text {eff }}, \log g$, and constant diffusion flux at all layers.

Each spectral line is calculated for at least 21 wavelength points starting with steps of $1 / 8$ the Doppler width at the line center, increasing gradually to also cover the broad damping wings. We have made some test calculations to test the dependence of the result on the number of spectral lines used. A typical example is the DA model at $T_{\text {eff }}=20000 \mathrm{~K}, \log g=8.00$ and $[\mathrm{Si} / \mathrm{H}]=$ -7.0 . With the 66 strongest lines of Si I to Si IV the change in the $\mathrm{Si}$ abundance at the same diffusion flux between calculations with vs. without radiative levitation is $0.23 \mathrm{dex}$ at $\tau_{\mathrm{R}}=2 / 3$, and 0.25 maximum considering all layers in the model between optical depth $10^{-6}$ and 1000 . Using $447 \mathrm{Si}$ lines, the numbers are 0.25 and 0.29 ; finally, with a large set of 824 lines the numbers are 0.23 and 0.32 . The major effect is already achieved with the small line set. If we want to answer the question whether radiative support without any current accretion is possible at the lowest abundance levels, we would need very high accuracy of the line absorption, and thus use as many lines as possible, because the answer is either yes or no. However, our main emphasis is to determine the diffusion fluxes, where a change of 0.05 dex is well within the typical errors. We thus decided to take the medium size set as a compromise between computing resources and accuracy. The corresponding calculations for C used 394 C I to $\mathrm{C}$ IV lines.

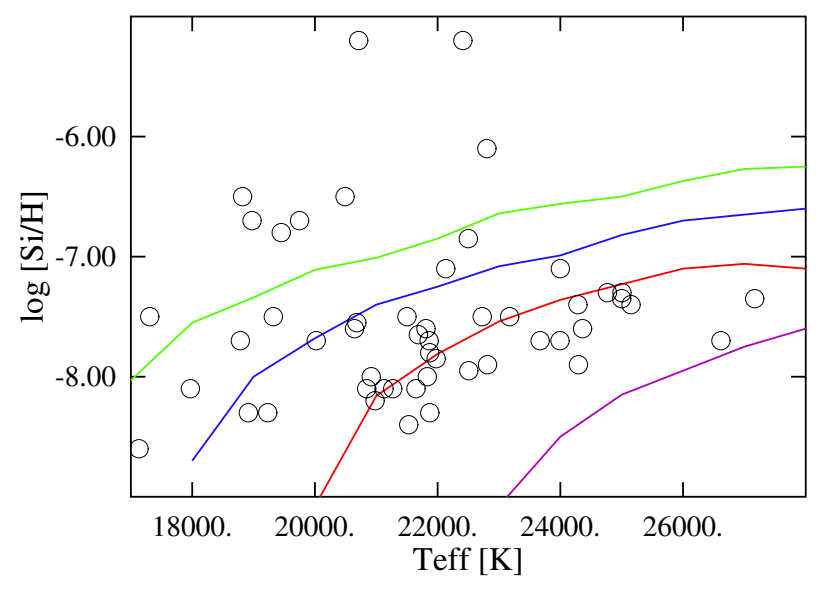

Fig. 5. Si abundances supported by radiative levitation without accretion. Continuous lines are the predictions for surface gravities $\log g=$ $7.50,7.75,8.00,8.25$ from top (green, blue, red, magenta in the color version). Small circles are the observed abundances from Table 1. The median $\log g$ of the stars with observed silicon is 7.94. See text for explanations.

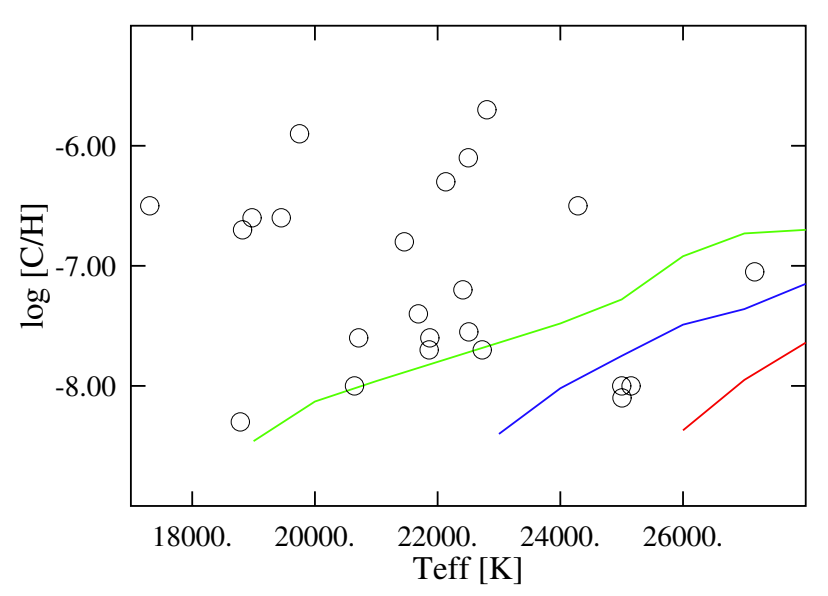

Fig. 6. Similar to Fig. 5, but for C. Continuous lines are the predictions for surface gravities $\log g=7.50,7.75,8.00$ from top (green, blue, red in the color version). The median $\log g$ of the stars with observed carbon is 7.90 .

To get an overview about the importance of radiative levitation we have calculated atmospheric models with the element stratification obtained with zero accretion flow. For these models synthetic spectra were calculated. In order to compare the results with the abundances in Table 1 we have then calculated homogenous models, which produced the same equivalent width as the stratified models. The abundances of these models are shown in Fig. 5 and compared to the Si abundances of Table 1. Figure 6 shows the same comparison for $\mathrm{C}$.

Figures 5 and 6 suggest that for a significant fraction of objects with photospheric Si radiative levitation may be important. On the other hand, for most of the $\mathrm{C}$ observations accretion seems to be necessary. The final answer is only possible by calculating in the next step the levitation for each object individually, using the appropriate stellar parameters from Table 1.

\subsection{Comparison with previous work}

Radiative levitation in white dwarfs has been calculated in several papers of the Montreal group (see e.g. Chayer et al. 1995a,b, and references therein) and by Dreizler \& Wolff (1999). These 
D. Koester et al.: The frequency of planetary debris around young white dwarfs

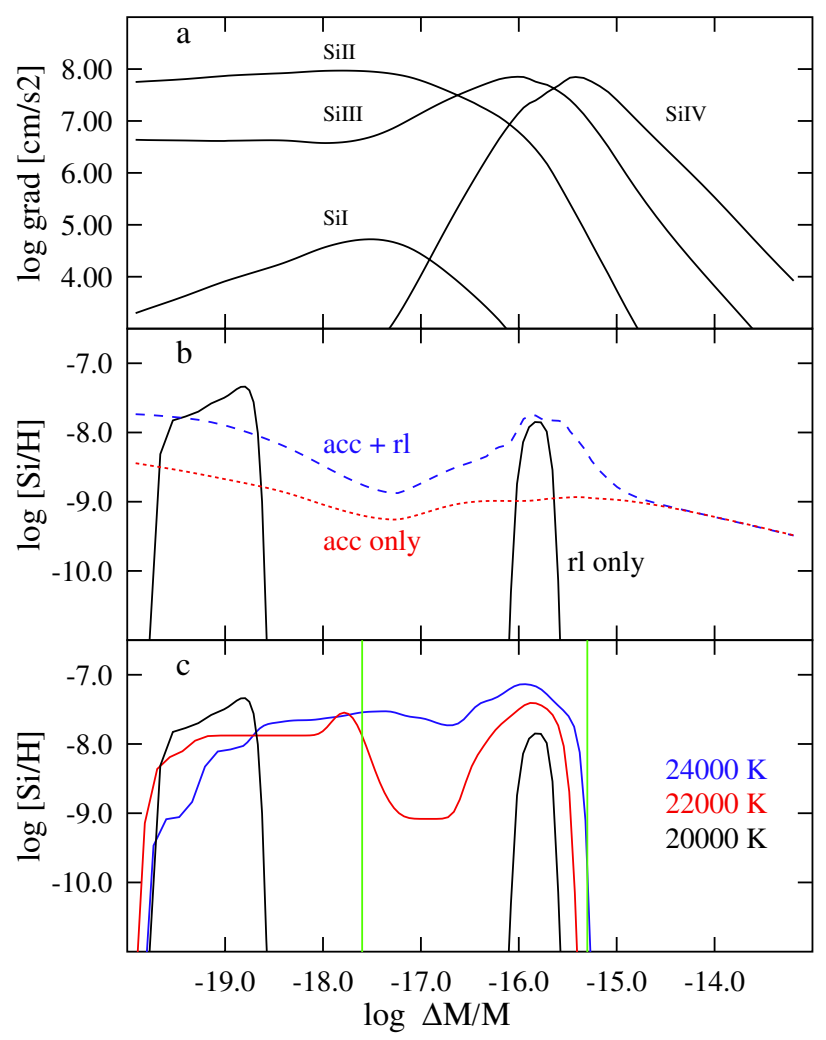

Fig. 7. Radiative support in a $\log g=8$ DA white dwarf model as a function of depth in the atmosphere, expressed as fractional mass $\Delta M / M$ from the surface.

studies were aimed at hot stars. We can, however, compare our results with the recent studies of Chayer (2014) and Chayer \& Dupuis (2010, hereafter C10). We use the same diffusion coefficients from Paquette et al. (1986), but the complete implementation and programming is independent. Chayer \& Dupuis (2010), give in their Fig. 3 the abundance distribution of Si in a $20000 \mathrm{~K}, \log g=8 \mathrm{DA}$ model for a range of accretion rates. Our own calculations for the same model are shown in Fig. 7. The four panels show:

a) Radiative support from the first four ions of silicon at $T_{\text {eff }}=$ $20000 \mathrm{~K}$, expressed as acceleration, weighted with the relative abundances of the ion.

b) Si abundance supported by this force. Without accretion two separate "clouds" of Si are formed; outside these clouds the abundance drops to zero (continuous black line). The dashed (blue) line shows the Si distribution when a small accretion/diffusion flux of $3.9 \times 10^{4} \mathrm{~g} \mathrm{~s}^{-1}$ is present, the dotted (red) line shows the same accretion flux with radiative levitation switched off.

c) Si abundance stratification for 24000,22000 , and $20000 \mathrm{~K}$ (from top on the right side). From 24000 to the upper limit of our sample near $27000 \mathrm{~K}$, the curves differ very little from the $24000 \mathrm{~K}$ curve. The main "visible" part of the atmosphere between $\tau_{\text {Rosseland }} 0.01-10$ is between the two vertical green lines.

The maximum abundance supported at the lowest accretion rates in $\mathrm{C} 10$ is $\log [\mathrm{Si} / \mathrm{H}] \approx-7.5$, which is very similar in our own calculations. However, there are significant differences between the two approaches

- C10 solve the time-dependent diffusion equation, whereas we determine the stationary state. We are interested in the interpretation of spectroscopic observations, which depend on the details of the atmosphere; according to Fig. 3 in $\mathrm{C} 10$ equilibrium is reached in less than one year. Any timedependent variations in the observed DAZ occur very likely on much longer time scales, justifying our use of the stationary state.

- C10 calculate envelope models. The atmospheric conditions at optical depth $2 / 3$ (corresponding to $\log \Delta M / M \approx-16.4$ in this model) are used as boundary conditions; the Si abundance is assumed to be constant above this layer. As the bottom panel of Fig. 7 demonstrates, the distribution of Si throughout the photosphere is very far from uniform (black continuous line). If we aim at a realistic comparison with observed spectra, we have to calculate synthetic spectra for this distribution, and it is quite clear in this case that the $\mathrm{Si}$ lines will be much weaker than in a model with constant $\log [\mathrm{Si} / \mathrm{H}]=-7.5$. We will come back to a specific example below.

- There is a qualitative difference between a model with zero accretion and one with even a very small accretion flux. If radiative support fails by a tiny amount to support $\mathrm{Si}$ at some layer in the atmosphere, the abundance will drop to zero without accretion. On the other hand, with accretion, the downward diffusion velocity can be very small, because of the near cancellation of the force terms in the diffusion equation. This will lead to a strong enhancement of the abundances as compared to regions without radiative support. This is demonstrated by the (blue) dashed line, which shows the Si distribution in the case of a small accretion flux $\left(3.9 \times 10^{4} \mathrm{~g} \mathrm{~s}^{-1}\right)$ and can be compared with the dotted (red) line, which shows the same accretion flux, but with radiative levitation switched off.

\section{Determination of diffusion fluxes for the sample with photospheric spectral lines}

Since radiative levitation depends strongly on the stellar parameters - especially the surface gravity - and the abundances, we need to study each object individually. For this purpose we calculated two sets of models for each set of atmospheric parameters; the first one without accretion, assuming equilibrium between radiative levitation and gravitational settling. For the resulting atmospheric structure a synthetic spectrum was calculated and from this the equivalent widths of the strong lines Si II $1265 \AA$ and C II $1335 \AA$ - and in a few cases additional lines - were calculated and compared with observed values. This includes obviously objects where no radiative support is possible, resulting in zero equivalent widths. The second set included accretion. The parameter we use in this calculation is the accretion flux (which in stationary state is also the diffusion flux). The flux for $\mathrm{Si}$ and $\mathrm{C}$ was varied, until a satisfactory fit was achieved; in the case of significant radiative support we determined an upper limit to the flux. Technically we used the original abundances from the fit with homogeneous models as a starting point. Assuming that they are approximately representative for the layer $\tau=2 / 3$, they lead, with the diffusion velocities calculated for this layer to a starting value for the flux.

Results of these calculations and the comparison with the observations are presented in Table 3. The column labeled R/A is our assessment, whether accretion is necessary to explain the observed line strengths or not. Unfortunately there is no simple and completely objective method for this decision. The strong dependence of radiative levitation on stellar parameters, and the 
Table 3. Equivalent widths (in m $\AA$ ) of Si II $1265 \AA$ and C II $1335 \AA$ measured from the observed spectra (obs) compared to the predictions of radiative levitation (RL).

\begin{tabular}{|c|c|c|c|c|c|c|c|c|}
\hline \multirow[t]{2}{*}{ Object } & \multicolumn{2}{|c|}{$E W(\mathrm{Si})[\mathrm{m} \AA]$} & \multicolumn{2}{|c|}{$E W(\mathrm{C})[\mathrm{m} \AA]$} & \multirow[t]{2}{*}{ Notes } & \multirow[t]{2}{*}{$\mathrm{R} / \mathrm{A}$} & \multicolumn{2}{|c|}{$\log$ flux $\left[\mathrm{g} \mathrm{s}^{-1}\right]$} \\
\hline & obs & RL & obs & RL & & & $\mathrm{Si}$ & $\mathrm{C}$ \\
\hline WD $0000+171$ & 54 & 30 & 0 & 0 & & $\mathrm{R}$ & $<4.20$ & $<4.02$ \\
\hline WD $0059+257$ & 291 & 7 & 47 & 0 & 1 & A & 6.13 & $<4.47$ \\
\hline WD $0102+095$ & 78 & 63 & 15 & 11 & & $\mathrm{R}$ & $<4.75$ & $<3.75$ \\
\hline WD 0114-605 & 88 & 80 & 25 & 31 & & $\mathrm{R}$ & $<4.48$ & $<3.48$ \\
\hline WD 0140-392 & 95 & 41 & 0 & 0 & & $\mathrm{R}$ & $<4.78$ & $<3.88$ \\
\hline WD 0242-174 & 56 & 16 & 0 & 0 & & $\mathrm{R}$ ? & $<4.03$ & $<4.23$ \\
\hline WD 0307+149 & 36 & 29 & 0 & 0 & & $\mathrm{R}$ & $<4.39$ & $<4.17$ \\
\hline WD $0341+021$ & 25 & 171 & 82 & 78 & & $\mathrm{R}$ & $<3.39$ & $<3.94$ \\
\hline WD $0352+018$ & 40 & 39 & & 0 & 5 & $\mathrm{R}$ & $<3.82$ & $<4.08$ \\
\hline HE0403-4129 & 90 & 36 & & 0 & 5 & $\mathrm{R}$ ? & $<5.34$ & $<4.35$ \\
\hline HE0416-1034 & 33 & 35 & 220 & 71 & 6 & A & $<4.70$ & 5.28 \\
\hline WD $0421+162$ & 144 & 0 & 0 & 0 & & A & 5.27 & $<4.49$ \\
\hline WD $0431+126$ & 55 & 2 & 0 & 0 & & A & 4.33 & $<4.36$ \\
\hline HE0452-3444 & 59 & 32 & 0 & 0 & & $\mathrm{R}$ & $<4.61$ & $<4.20$ \\
\hline HS0507+0434A & 20 & 5 & 0 & 0 & & $\mathrm{R}$ ? & $<3.91$ & $<4.12$ \\
\hline WD $0710+741$ & 245 & 118 & 277 & 10 & $*, 1,2,6$ & A & 5.39 & 6.51 \\
\hline WD $0843+516$ & 126 & 0 & 90 & 0 & 1,4 & A & 7.56 & 5.38 \\
\hline WD 0854+404 & 41 & 58 & & 0 & 5,7 & $\mathrm{R}$ & $<4.15$ & $<4.19$ \\
\hline WD $0920+363$ & 54 & 112 & & 54 & 5 & $\mathrm{R}$ & $<4.35$ & $<3.68$ \\
\hline WD 0954+697 & 49 & 40 & 0 & 0 & & $\mathrm{R}$ & $<4.08$ & $<4.23$ \\
\hline WD $1013+256$ & 159 & 28 & 141 & 0 & & A & 5.52 & 6.28 \\
\hline WD 1015+161 & 409 & 0 & 0 & 0 & 1,4 & A & 6.54 & $<4.84$ \\
\hline WD $1017+125$ & 98 & 34 & & 0 & 5 & $\mathrm{R}$ ? & $<4.86$ & $<4.94$ \\
\hline WD 1034+492 & 123 & 0 & 0 & 0 & & A & 5.17 & $<4.77$ \\
\hline WD $1038+633$ & 39 & 3 & & 0 & 5,7 & $\mathrm{~A}$ & 4.69 & $<4.59$ \\
\hline WD $1129+155$ & 30 & 0 & & 0 & 5 & A & 4.55 & $<4.76$ \\
\hline WD $1133+293$ & 50 & 75 & & 4 & 5 & $\mathrm{R}$ & $<4.23$ & $<4.38$ \\
\hline SDSS1228+1040 & 1090 & 0 & 48 & 0 & $*, 1,4,5$ & A & 7.72 & 5.23 \\
\hline HS1243+0132 & 51 & 48 & & 0 & 5 & $\mathrm{R}$ & $<3.19$ & $<4.00$ \\
\hline WD $1257+048$ & 91 & 39 & 38 & 0 & & A & 4.80 & 4.71 \\
\hline WD 1310-305 & 96 & 15 & & 0 & 5 & A & 4.70 & $<4.51$ \\
\hline WD $1325+279$ & 34 & 19 & & 0 & 5 & $\mathrm{R}$ & $<4.25$ & $<4.36$ \\
\hline WD 1325-089 & 29 & 0 & & 0 & 5 & A & 4.20 & $<4.75$ \\
\hline WD $1408+323$ & 121 & 0 & 18 & 0 & & $\mathrm{~A}$ & 4.95 & 4.42 \\
\hline WD 1451+006 & 27 & 54 & & 32 & 5 & $\mathrm{R}$ & $<4.01$ & $<3.02$ \\
\hline WD $1548+149$ & 69 & 40 & 61 & 0 & & A & 4.66 & 5.09 \\
\hline WD $1647+375$ & 339 & 59 & 218 & 0 & 1 & $\mathrm{~A}$ & 6.44 & 7.06 \\
\hline WD $1713+332$ & 37 & 145 & 58 & 60 & & $\mathrm{R}$ & $<3.45$ & $<4.26$ \\
\hline WD $1755+194$ & 71 & 72 & 19 & 20 & & $\mathrm{R}$ & $<4.77$ & $<3.61$ \\
\hline WD 1914-598 & 19 & 12 & & 0 & 5 & $\mathrm{R}$ & $<3.97$ & $<4.31$ \\
\hline WD 1929+012 & 1559 & 41 & 130 & 0 & $*, 1,4$ & A & 8.03 & 5.85 \\
\hline WD $1943+163$ & 271 & 7 & 139 & 0 & 1 & A & 5.84 & 6.10 \\
\hline WD 1953-715 & 314 & 0 & 141 & 0 & 1 & A & 6.06 & 6.16 \\
\hline WD 2046-220 & 76 & 56 & & 0 & 5 & $\mathrm{R}$ & $<4.46$ & $<4.52$ \\
\hline WD $2058+181$ & 156 & 0 & 135 & 0 & 1 & A & 5.37 & 6.28 \\
\hline WD $2134+218$ & 67 & 0 & & 0 & 5 & A & 4.89 & $<4.70$ \\
\hline HS2229+2335 & 350 & 0 & 125 & 0 & & A & 6.35 & 6.09 \\
\hline HE2231-2647 & 36 & 48 & & 0 & 5 & $\mathrm{R}$ & $<3.88$ & $<3.98$ \\
\hline HS2244+2103 & 32 & 45 & & 0 & 5 & $\mathrm{R}$ & $<4.35$ & $<3.95$ \\
\hline WD $2256+249$ & 145 & 74 & 168 & 0 & $*, 1,2$ & A & 5.49 & 6.03 \\
\hline WD $2257+162$ & 66 & 141 & 110 & 74 & $*, 2$ & $\mathrm{R}$ & $<3.48$ & $<5.58$ \\
\hline WD $2306+124$ & 120 & 0 & 20 & 0 & & A & 4.98 & 4.70 \\
\hline WD 2322-181 & 84 & 23 & 47 & 0 & & A & 4.67 & 4.86 \\
\hline
\end{tabular}

Notes. Logarithms of the $\mathrm{C}$ and Si diffusion fluxes (in $\mathrm{g} \mathrm{s}^{-1}$ ). See Sect. 7, 8 for details. *: ancillary target, not used in sample statistics; 1: additional elements detected; 2: post-common envelope (close) binary; 3: spatially resolved (wide) binary; 4: exhibits excess infrared emission; 5: C II 1335 line contaminated by interstellar line; if field is empty, the spectrum is consistent with a photospheric $E W=0$; 6: C III lines near $1175 \AA$ used instead of $1335 \AA$; 7: Si II $1298 \AA$ A line used instead of $1265 \AA$

unavoidable uncertainties of these parameters, as well as uncertainties of the equivalent widths for very weak lines and noisy spectra, lead to an intermediate range without unambiguous decisions. We have aimed to be conservative and rather err on the assignment of $\mathrm{R}$ than $\mathrm{A}$, that is, we want to be confident that the latter objects are really accreting. If the predicted EWs are within a factor of two of the observed ones, we consider pure radiative support (without accretion) as possible, whereas with 
a factor of four or larger we conclude ongoing accretion. In between, the decision was made using the quality of the spectra, and if no decision seemed possible, the assignment was $\mathrm{R}$ ?

With this classification, we conclude that of the 48 objects showing photospheric $\mathrm{Si}, 23$ must be currently accreting. Likewise, of the 19 objects with C, 14 must be accreting. There is one single object in Table 3 (HE0416-1034), where the $\mathrm{Si}$ abundance could be explained by radiative levitation alone, but the $\mathrm{C}$ abundance apparently demands accretion. Other results of Table 3 are further discussed in Sect. 9.

There has been a recent claim by Deal et al. (2013) that the diffusion fluxes would change by orders of magnitude, if the thermohaline instability would be taken into account. In Xu et al. (2014) we discuss some arguments, why we do not think that these calculation apply to the diffusion in white dwarfs. In that paper, we were presenting results for white dwarfs with an outer convection zone. It is conceivable that at the bottom of the convection zone a boundary layer develops with a discontinuity in molecular weight, if the accretion of a large amount of external matter (of asteroid size) is very fast, compared to diffusion timescales. In the current sample the stars do not have any convection zone and we believe that the Deal et al. (2013) calculations are irrelevant in this case. A more detailed discussion will be given in a future paper (Koester, in prep.).

\subsection{Effect of parameter errors on diffusion calculations}

We use the same object as above (WD 1943+163) to estimate the change in the diffusion fluxes within the error estimates for $T_{\text {eff }}$ and $\log g$. With unchanged abundances, the diffusion flux changes for all elements by approximately $0.05 \mathrm{dex}$, in the same direction that the abundances have to change in the spectral analysis. So the combined uncertainty of the atmospheric parameters on the final diffusion fluxes can be estimated as 0.09 dex. This should be added quadratically to the (statistical) abundance uncertainties to get errors for the individual fluxes. However, since the changes go into the same direction for all elements, these errors need not be added when calculating element ratios.

\section{Results and discussion}

Taken at face value the number of metal-polluted white dwarfs in the COS sample (48 out of 85 , or $56 \%$, see Table 2 ) is surprisingly high. Previous estimates (e.g. Zuckerman et al. 2003, 2010) put this number much lower at 20-30\%. Out of the 48 stars with at least a detection of photospheric Si, 23 (27\%) must be currently accreting, and in an additional 25 (29\%), the Si abundance is compatible with radiative levitation, however, still implying accretion in the recent past (see Sect. 9.1 below). This increased fraction of metal-pollution among our COS sample compared to previous optical studies is probably due to the relatively high resolution and signal-to-noise ratio of our observations, and to the choice of the wavelength region with strong lines of $\mathrm{Si}$, a major component of planetary debris. Different from previous work on larger samples (Zuckerman et al. 2003; Koester et al. 2005, 2009) our sample was specifically chosen for a search for metal traces in fairly bright stars in a limited and well defined temperature range. The detection threshold for $\mathrm{Si}$ does not significantly vary over this temperature range (see the solid line in the bottom panel of Fig. 8), and we are confident that the high fraction of metal-polluted white dwarfs is representative for the local neighborhood within $\approx 100$ pc around the Sun.

\subsection{Accretion from the interstellar medium?}

Twenty five (29\%) of the stars in our sample have Si abundances that are consistent with being supported by radiative levitation what is the nature, and the origin of the polluting material? Figure 8 in Chayer et al. (1995b) suggests that the equilibrium abundance of Si supported by radiative levitation reaches a broad minimum around $T_{\text {eff }} \approx 70000 \mathrm{~K}$ along the cooling sequence for a $0.6 M_{\odot}$ white dwarf (corresponding to a cooling age of $<1 \mathrm{Myr})$. Upon further cooling to $30000 \mathrm{~K}(\approx 10 \mathrm{Myr})$, the abundance necessary for equilibrium rises by a factor of $\approx 100$. At abundances lower than the equilibrium values - as those possibly remaining from the earlier evolution - radiative levitation will always dominate gravitational settling. Our interpretation is that any remaining primordial Si would be blown out of the star by the radiation pressure. As a conclusion, any $\mathrm{Si}$ visible below $30000 \mathrm{~K}$ must have been accreted. Before claiming that this must always be accretion from a remnant planetary system or circumstellar material in general, we derive some estimates concerning the possibility of accretion from the interstellar medium.

The total $\mathrm{Si}$ mass in radiative levitation equilibrium in the atmosphere of a $20000 \mathrm{~K}, \log g=8 \mathrm{DA}$ is $7.89 \times 10^{10} \mathrm{~g}$ (Fig. 7). The cross section for Eddington accretion from interstellar matter, which is the minimum we would expect, is

$a=\frac{2 \pi G M_{\mathrm{wd}}}{v^{2}} R_{\mathrm{wd}}=5.29 \times 10^{22} \mathrm{~cm}^{2}$

with white dwarf mass and radius $M_{\mathrm{wd}}$ and $R_{\mathrm{wd}}$, gravitational constant $G$ and space velocity $v$. Assuming the white dwarf crosses just one tiny cloud like our Local Interstellar Cloud (LIC) with a Si column density of $10^{13} \mathrm{~cm}^{-2}$ and a velocity of $30 \mathrm{~km} \mathrm{~s}^{-1}$ (Redfield \& Linsky 2004), it would sweep up $2.46 \times 10^{13} \mathrm{~g}$ of Si. That is three hundred times more than needed to explain the observations, and most of this matter would diffuse downward, except for the tiny fraction supported by radiative levitation. However, in this scenario, adopting a typical cloud size of $\approx 2$ pc within the solar neighborhood (Redfield \& Linsky 2004), the accretion rate of $\mathrm{Si}$ would be $\dot{M}(\mathrm{Si})<10 \mathrm{~g} \mathrm{~s}^{-1}$, orders of magnitude smaller than the rates determined for the 23 objects with inferred current accretion. A more realistic estimate for the accretion of Si bound in interstellar dust grains is obtained assuming that hydrodynamic (Bondi-Hoyle) accretion works only within a radius, where the grains sublimate (Alcock \& Illarionov 1980; Farihi et al. 2010a). A similar calculation as above leads to accretion rates $\dot{M}(\mathrm{Si})<10^{3} \mathrm{~g} \mathrm{~s}^{-1}$, still much smaller than any of our observed rates.

An additional argument against an ISM origin for the Si detections in our sample comes from the $\mathrm{C} / \mathrm{Si}$ ratio that we determined for the debris material. Accretion from the ISM would suggest a $\mathrm{C} / \mathrm{Si}$ ratio close to its solar value, i.e. $\approx 3.6$ by mass. Inspection of Table 3 shows that the majority of the white dwarfs that are currently accreting have $\mathrm{C} / \mathrm{Si}$ values, or upper limits thereof, significantly below solar. Exceptions are the white dwarfs in post-common envelope binaries, which presumably accrete $\approx$ solar-abundance wind from their companions, plus a handful of apparently single white dwarfs, the nature of which which will be discussed elsewhere.

\subsection{Total accretion rates}

Given that typically only one, or at best a few, elements are detected in the white dwarf photosphere, computing the total accretion must rely on an assumption regarding the chemical 


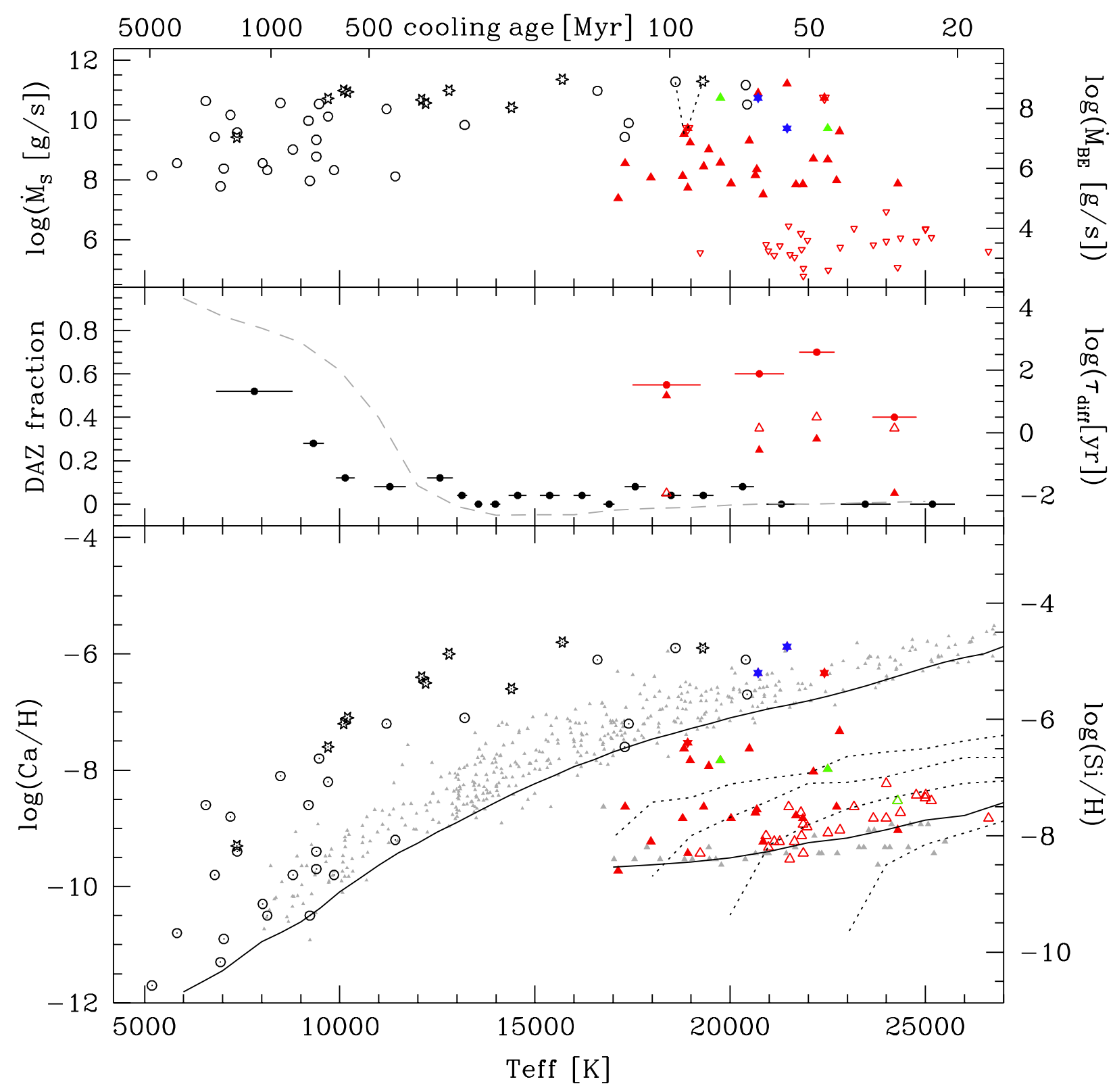

Fig. 8. Metal pollution in DA white dwarfs as a function of $T_{\text {eff }}$ (bottom axis) and cooling age (top axis, for log $g=8$ ). Star-shaped symbols represent white dwarfs where their dust disks were detected in the infrared. Bottom panel: $\log (\mathrm{Ca} / \mathrm{H})$ abundances (open circles and stars) and upper limits (gray dots) from Koester \& Wilken (2006). Middle panel: the fraction of white dwarfs with photospheric Ca and Si detections. Top panel: inferred total accretion rates, where the $\mathrm{Si}$ and $\mathrm{Ca}$ abundances have been scaled by bulk Earth abundances $\left(\dot{M}_{\mathrm{BE}}\right)$ and by solar abundances $\left(\dot{M}_{\mathrm{S}}\right)$. See text for further explanations.

composition of the accreting material. Historically, solar abundances were adopted within the context of accretion from the interstellar medium, see e.g. Koester \& Wilken (2006) for a discussion of deriving total accretion rates from the photospheric $\mathrm{Ca}$ abundances. Over the past decade, it has become increasingly clear that planetary debris is the most likely origin of the metal-pollution detected in a large number of white dwarfs, and, given that overall the chemical abundance patterns bear a strong resemblance to rocky solar system material, using bulk Earth abundances to infer total accretion rates has become a standard assumption - again, most of the times extrapolated from the photospheric Ca abundances (e.g. Farihi et al. 2009; Zuckerman et al. 2010; Girven et al. 2012).
Figure 8 summarizes many of our results. The bottom panel shows $\log (\mathrm{Ca} / \mathrm{H})$ abundances (open circles and stars) and upper limits (gray dots) from Koester \& Wilken (2006) (compiled from Zuckerman et al. 2003; Berger et al. 2005). Red symbols illustrate the $\log (\mathrm{Si} / \mathrm{H})$ abundances from our HST/COS survey, where filled red symbols represent the 23 stars that must currently be accreting (labeled " $\mathrm{A}$ " in Table 3), open triangles are the 25 stars where $\mathrm{Si}$ is detected close to the equilibrium abundance for radiative levitation (labeled "R" and "R?" in Table 3), and upper limits to $\log (\mathrm{Si} / \mathrm{H})$ for the remaining 37 stars are shown by small gray triangles. For comparison, the parameters of five additional DA white dwarfs that we observed with COS (Gänsicke et al. 2012) are shown in blue (the debris-accreting 
SDSS $1228+1040$ and WD 1929+019) and green (the three PCEBs WD 0710+741, WD 2256+249, WD 2257+162). These five stars are not included in the discussion of the COS DA sample statistics (Sect. 9.4 and middle panel of this figure). Infrared excesses from circumstellar dust have been observed at four stars observed with COS (blue: SDSS 1228+1040 and WD 1929+019, red: WD 0843+516 and WD 1015+161). The $\log (\mathrm{Ca} / \mathrm{H})$ and $\log (\mathrm{Si} / \mathrm{H})$ axes are offset by the bulk Earth $\log (\mathrm{Si} / \mathrm{Ca})=-1.128(\mathrm{McDonough} 2000)$. The solid lines correspond to the typical detection limits of $\mathrm{Ca}$ achieved by highresolution ground-based spectroscopy $(E W \approx 15 \mathrm{~m} \AA$ in the $\mathrm{CaK}$ line), and of $\mathrm{Si}$ in our HST/COS observations $(E W \approx$ $30 \mathrm{~m} \AA$ in Si II $1265 \AA$ ). The four dashed lines give the Si equilibrium abundance for $\log g=7.50,7.75,8.00$, and 8.25 (from top to bottom).

In the middle panel the fraction of white dwarfs with photospheric $\mathrm{Ca}$ and $\mathrm{Si}$ detections is shown. Each black dot represents 25 stars with Ca measurements, with the error bar given by the standard deviation in $T_{\text {eff }}$. Each red dot represents 20 stars with $\mathrm{Si}$ measurements from our COS survey, which are further sub-divided into white dwarfs that are currently accreting (filled triangles), and in which photospheric Si could be maintained by radiative levitation (open triangles). The gray dashed line illustrates the diffusion time scale for $\mathrm{Ca}$ at $\tau=2 / 3$ in radiative atmospheres, and at the bottom of the convection zone in convective atmospheres, the transition between the two regimes occurs between $12000 \mathrm{~K}$ and $13000 \mathrm{~K}$.

The top panel of Fig. 8 shows the total accretion rates for the 38 DAZ from Koester \& Wilken (2006), for the 25 accreting DAZ from our COS survey, and for five additional objects that we observed for comparison (two white dwarfs with infrared excess from their circumstellar debris disks, and three post-common envelope binaries - these objects are not used for the statistics discussed below). For the stars observed with COS, we scaled the Si mass fluxes (Table 3) with the Si mass fraction for bulk-Earth and solar abundances, 0.161 (McDonough 2000) and $6.65 \times 10^{-4}$ (Asplund et al. 2009), respectively. Open circles are from Table 3 in Koester \& Wilken (2006), based on the extrapolation from the photospheric $\mathrm{Ca}$ abundances, the filled/colored symbols are extrapolated from the photospheric $\mathrm{Si}$ accretion fluxes determined from our COS spectra. Open red triangles show upper limits from our COS survey. Two stars are common to our COS survey and the Koester \& Wilken (2006) study (WD1015+161, HS2229+2335), their accretion rates extrapolated from $\mathrm{Ca}$ and $\mathrm{Si}$ are joined by dotted lines, and illustrate the uncertainty in $\dot{M}$ extrapolated from just one element, given that the accreting material can show large deviations from the solar or bulk Earth Si/Ca ratio (see Fig. 7 in Gänsicke et al. 2012).

While the abundances of planetary debris are broadly speaking "rock-like" (i.e. volatile depleted and rich in $\mathrm{O}, \mathrm{Si}, \mathrm{Mg}$, $\mathrm{Fe})$, we have shown that there are substantial variations in the metal-to-metal abundance ratios (Gänsicke et al. 2012), which implies that total accretion rates based on either $\mathrm{Ca}$ or $\mathrm{Si}$ can easily diverge by an order of magnitude. WD 1015+161 and HS 2229+2335 are both in our COS sample, and among the DAZ of Koester \& Wilken (2006), in both cases the total accretion rate determined from the photospheric $\mathrm{Ca}$ abundance significantly exceeds that based on our Si measurement. However, judging from Table 4 and Fig. 7 in Gänsicke et al. (2012), it is clear that WD 1015+161 has overall a much lower Si abundance compared to the other stars. The case is similar for HS 2229+2335, where we computed a Ca flux of $10^{7.15} \mathrm{~g} \mathrm{~s}^{-1}$ (using $\log [\mathrm{Ca} / \mathrm{H}]=-5.9$ from Koester \& Wilken 2006). For white dwarfs with such high temperatures, ground-based spectroscopy is only sensitive to the highest $\mathrm{Ca}$ abundances, and as such it is maybe not surprising that both WD 1015+161 and HS 2229+2335 have unusually high $\mathrm{Ca} / \mathrm{Si}$ ratios. More accurate total accretion rates can only be derived if all the main elements are detected, i.e. at least $\mathrm{O}, \mathrm{Mg}$, $\mathrm{Si}$, and $\mathrm{Fe}$ for rocky material, plus $\mathrm{C}$ in the case of volatile-rich material (see Farihi et al. 2012b for a quantitative comparison).

Taking the accretion rates shown in Fig. 8 at face value, it is evident that the ranges of rates probed by searches for $\mathrm{Ca}$ and $\mathrm{Si}$ at low and high $T_{\text {eff }}$ are entirely complementary, and lead to a broadly similar distribution. Adopting bulk Earth abundances for the accreted material, the measured accretion rates range from few $10^{5} \mathrm{~g} \mathrm{~s}^{-1}$, set by the detection threshold of the observations, to a few $10^{8} \mathrm{~g} \mathrm{~s}^{-1}$. This upper limit agrees, within an order of magnitude, with the accretion rate that would be driven purely by Poynting-Robertson drag on dust particles near the inner edge of the debris disk (Rafikov 2011a).

However, sublimation of the dust will unavoidably lead to the additional presence of gas within the disk, and the gas content may be enhanced e.g. by the impact of additional asteroids on an existing debris disk (Jura 2008). This gaseous phase has been detected both from emission lines arising in the outer parts of the disk (Gänsicke et al. 2006, 2007, 2008; Farihi et al. 2012a; Melis et al. 2012) and absorption lines along the line of sight onto the white dwarf (Debes et al. 2012; Gänsicke et al. 2012). The additional viscosity of this gas is expected to increase the accretion rate over the value for Poynting-Robertson alone, potentially leading to a runaway process with peak rates of $10^{10}-10^{11} \mathrm{~g} \mathrm{~s}^{-1}$ (Rafikov 2011b; Metzger et al. 2012). Such high accretion rates are observationally inferred for a number of DBZ white dwarfs (Farihi et al. 2012b; Girven et al. 2012). However, because of the long diffusion time scales in these stars, they are most likely not in accretion-diffusion equilibrium, and the accretion rates derived for them should be interpreted as a long-term average value - which implies that the peak accretion rates are probably even higher. The absence of any DAZ stars with accretion rates $\left(\dot{M}>10^{10} \mathrm{~g} \mathrm{~s}^{-1}\right)$ strongly suggests that such phases have short life times, and correspondingly small probabilities of being detected.

A final note concerns the overall distribution of accretion rates. Ignoring the uncertainties on $\dot{M}$ for any individual system, it appears that there is very little dependence of the derived accretion rates on the cooling age of the stars, which ranges from a few 10 Myr at the hot end to $\approx 2$ Gyr at the cool end. There is some lack of white dwarfs with low accretion rates in the range $12000 \mathrm{~K} \lesssim T_{\text {eff }} \lesssim 17000 \mathrm{~K}$, but this is possibly caused by the decreasing sensitivity of ground-based spectroscopy for photospheric $\mathrm{Ca} H \& \mathrm{~K}$ lines. However, at $T \gtrsim 23000 \mathrm{~K}$, there is a sudden drop in stars with high accretion rates - the COS spectroscopy remains extremely sensitive to $\mathrm{Si}$ at these temperatures, so this deficiency is real, and its cause is discussed below in Sect. 9.4.

\subsection{Objects without photospheric Si}

Radiative levitation predicts detectable amounts of Si over most of the temperature range of our sample. Why do 37 stars show no trace of $\mathrm{Si}$ or $\mathrm{C}$ ? In Table 4 we show the predicted equivalent widths of the Si II $1265 \AA$ and C II $1335 \AA$ lines. The fourth column (Detectable? N/Y) is our estimate if these predicted lines should be visible or not, given the individual quality of the COS spectra. We would expect to detect metals only in 8 
Table 4. Predicted equivalent widths (EW in mA) of Si II $1265 \AA$ and C II $1335 \AA$ for objects where no metal pollution is detected in their COS spectra.

\begin{tabular}{|c|c|c|c|}
\hline Object & $\begin{array}{r}E W(\mathrm{Si}) \\
{[\mathrm{m} \AA]}\end{array}$ & $\begin{array}{r}E W(\mathrm{C}) \\
{[\mathrm{m} \AA]}\end{array}$ & Detectable? \\
\hline WD 0013-241 & 0 & 0 & $\mathrm{~N}$ \\
\hline WD 0018-339 & 29 & 0 & $\mathrm{~N}$ \\
\hline WD 0028-474 & 0 & 0 & $\mathrm{~N}$ \\
\hline WD 0047-524 & 0 & 0 & $\mathrm{~N}$ \\
\hline WD 0124-257 & 75 & 12 & $\mathrm{Y}$ \\
\hline WD $0155+069$ & 54 & 0 & $\mathrm{Y}$ \\
\hline HS0200+2449 & 13 & 0 & $\mathrm{~N}$ \\
\hline HE0308-2305 & 0 & 0 & $\mathrm{~N}$ \\
\hline WD $0308+188$ & 0 & 0 & $\mathrm{~N}$ \\
\hline HE0358-5127 & 9 & 0 & $\mathrm{~N}$ \\
\hline HE0414-4039 & 7 & 0 & $\mathrm{~N}$ \\
\hline HE0418-1021 & 0 & 0 & $\mathrm{~N}$ \\
\hline WD $0933+025$ & 45 & 0 & $\mathrm{~N}$ \\
\hline HS0944+1913 & 0 & 0 & $\mathrm{~N}$ \\
\hline WD $0947+325$ & 0 & 0 & $\mathrm{~N}$ \\
\hline WD $1005+642$ & 5 & 0 & $\mathrm{~N}$ \\
\hline WD 1049-158 & 0 & 0 & $\mathrm{~N}$ \\
\hline WD $1049+103$ & 24 & 0 & $\mathrm{~N}$ \\
\hline WD $1052+273$ & 0 & 0 & $\mathrm{~N}$ \\
\hline WD 1058-129 & 0 & 0 & $\mathrm{~N}$ \\
\hline WD $1102+748$ & 0 & 0 & $\mathrm{~N}$ \\
\hline WD $1104+602$ & 0 & 0 & $\mathrm{~N}$ \\
\hline WD 1229-013 & 112 & 29 & $\mathrm{Y}$ \\
\hline WD 1230-308 & 28 & 0 & $\mathrm{~N}$ \\
\hline WD $1330+473$ & 48 & 0 & $\mathrm{Y}$ \\
\hline WD $1353+409$ & 128 & 60 & $\mathrm{Y}$ \\
\hline WD $1459+347$ & 0 & 0 & $\mathrm{~N}$ \\
\hline WD 1524-749 & 91 & 30 & $\mathrm{Y}$ \\
\hline WD 1531-022 & 0 & 0 & $\mathrm{~N}$ \\
\hline WD $1547+057$ & 0 & 0 & $\mathrm{~N}$ \\
\hline WD $1633+676$ & 34 & 0 & $\mathrm{~N}$ \\
\hline WD 2021-128 & 21 & 0 & $\mathrm{~N}$ \\
\hline WD $2032+188$ & 59 & 4 & $\mathrm{Y}$ \\
\hline HS2210+2323 & 0 & 0 & $\mathrm{~N}$ \\
\hline WD $2220+133$ & 0 & 0 & $\mathrm{~N}$ \\
\hline HE2238-0433 & 0 & 0 & $\mathrm{~N}$ \\
\hline WD 2359-324 & 70 & 22 & $\mathrm{Y}$ \\
\hline
\end{tabular}

Notes. In the third column Y means the line should be visible, $\mathrm{N}$ the opposite.

of the objects. Two might indeed show some Si, but the spectrum is strongly perturbed near the $1265 \AA$ line (WD 1229-013, WD 1230-308). For the six remaining stars, there is no obvious reason why no metals are detected, and our models indicate these systems lack a source of external pollution. Even in the case where $100 \%$ of stars form planetary systems, this alone is insufficient to deliver Si to the surfaces of all their white dwarf remnants within the appropriate timescale. Successful models that deliver debris to the surfaces of white dwarfs indicate that a combined planet-planetesimal belt is necessary (Bonsor et al. 2011; Debes et al. 2012) and it seems plausible that this architecture may be common but not universal.

Closer inspection of the atmospheric parameters reveals a significant difference between the stars where no metals are detected, and those where metals are observed at abundances compatible with radiative levitation (Table 3), which is illustrated in Fig. 9: the latter objects are concentrated in the lower right corner, at high temperatures and low surface gravities. Given the mechanism of radiative levitation this is exactly what is

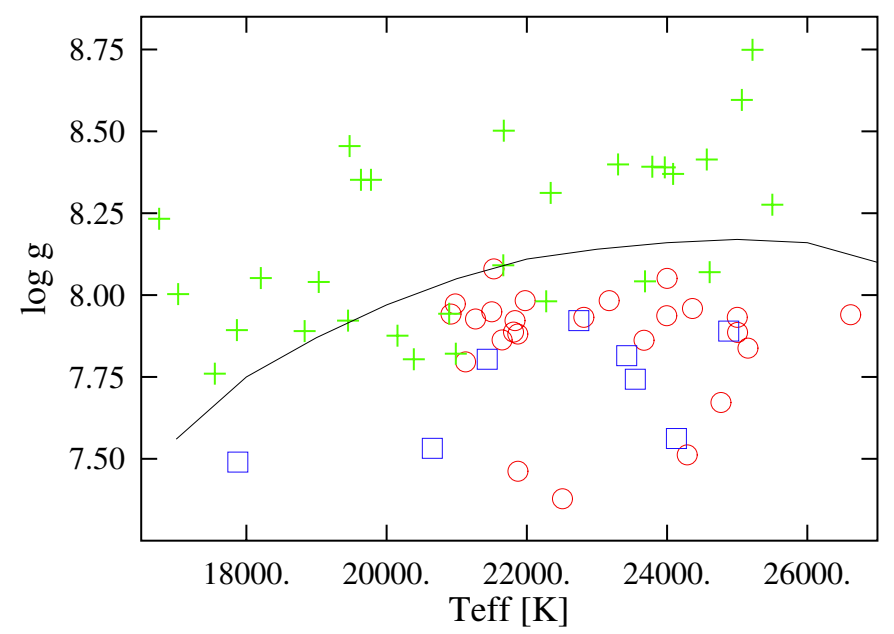

Fig. 9. Objects where $\mathrm{Si}$ is detected at levels consistent with radiative levitation (red circles), and objects where $\mathrm{Si}$ is neither detected, nor expected to be radiatively supported for their $T_{\text {eff }}$ and $\log g$ (green crosses). The blue squares indicate the parameters of eight stars where radiative levitation is strong enough to produce visible lines, yet, no metals are detected in their COS spectra. The black continuous line indicates the location for an EW of $30 \mathrm{~m} \AA$ of the Si II 1265 line, which is our detection threshold.

expected, in objects with lower temperatures and/or higher gravity, $\mathrm{Si}$ sinks out of the photosphere. The continuous line indicating the combination of $T_{\text {eff }}$ and $\log g$ where radiative levitation results in $30 \mathrm{~m} \AA$ equivalent widths, which is our typical detection threshold, neatly fits to the division of the two sets of stars. This is a strong indication that our calculations are overall correct.

\subsection{The fraction of white dwarfs with remnants of planetary systems}

While the total fraction of Si-polluted white dwarfs in our sample is relatively independent of $T_{\text {eff }}$, hovering at $\approx 50 \%$ (red points in the middle panel of Fig. 8), the distribution of white dwarfs that must be currently accreting and those where the metal pollution can be explained by the equilibrium abundance obtained from the radiative levitation calculations show a very distinct pattern. At temperatures below $\approx 20000 \mathrm{~K}$, radiative levitation becomes very ineffective, and correspondingly the vast majority of stars with Si detections must be currently accreting in other words, the fraction of accreting white dwarfs is equal to the fraction of stars with Si detections, $\approx 50 \%$. Towards higher temperatures, this pattern reverses, and the majority of Si detections are consistent with radiative levitation alone. In addition, as already noted in the previous section, there is a striking lack of stars with high accretion rates at $T_{\text {eff }} \gtrsim 23000 \mathrm{~K}$.

As argued in Sect. 9.1, planetary debris is the only plausible source of metals for the 25 stars that are currently accreting. Looking at Fig. 8, these stars have cooling ages of $\approx 40-100 \mathrm{Myr}$, in contrast, at younger cooling ages we find only stars with relatively low photospheric $\mathrm{Si}$ abundances which can be explained by radiative levitation. Yet, as shown in Sect. 9.1, these stars also must have accreted at some point in the past. A priori, and dynamically, there is no reason why ongoing accretion of planetary debris should become suddenly more frequent at white dwarfs with cooling ages older than $40 \mathrm{Myr}$.

One plausible explanation for this dichotomy relies on the fact that observing a white dwarf in a phase of ongoing accretion 
requires the availability of a reservoir of material. Such a reservoir has been found in the form of dusty and gaseous disks around 30 white dwarfs (e.g. Zuckerman \& Becklin 1987; Gänsicke et al. 2006; Farihi et al. 2009; Brinkworth et al. 2012). While strongly constrained only for about $1 / 3$ of known dusty white dwarfs that were observed with Spitzer out to $24 \mu$, the outer radii of these disks are compatible with the tidal disruption radius (Roche limit) of a typical white dwarf, $\approx 1 R_{\odot}$. Their inner radii are consistent with the distance from the white dwarf at which the radiation field is sufficiently strong to sublimate the dust. Hence, the location of the inner disk radii depends on the composition of the dust and its typical grain sizes (which determines the sublimation temperature), and to a greater extent on the white dwarf luminosity. While the dust parameters are somewhat uncertain, it is straightforward to see that the inner edge of the disk moves out with increasing $T_{\text {eff }}$. Von Hippel et al. (2007) and Farihi (2011) showed that, adopting a range of assumptions on grain size and sublimation temperature, the inner (sublimation) radius of a dusty debris disk will become equal to the Roche limit somewhere in the range 15000-22000 K. More recently, Rafikov \& Garmilla (2012) showed that the sublimation temperatures of Si-rich minerals in a H-deficient environment are a few $100 \mathrm{~K}$ higher than the typically adopted values (valid in H-rich conditions, such as protoplanetary disks), suggesting that the upper end of that temperature range is more realistic.

The implication of all the above is that while planetesimal disruption events can and probably do occur as frequent at white dwarfs hotter than $\approx 23000 \mathrm{~K}$, and dynamically this is actually favored at earlier cooling ages (Debes \& Sigurdsson 2002; Bonsor \& Wyatt 2011; Veras et al. 2013; Frewen \& Hansen 2014), the debris will rapidly sublimate, forming a purely gaseous disk. Given that the viscosity of gas is much higher than that of dust, angular momentum transfer in a gaseous disk is substantially more efficient, dramatically reducing the life time of a gaseous disk compared to the dust disks that form around cooler white dwarf. Once the debris of the tidal disruption event is delivered onto the white dwarf, the small amount of $\mathrm{Si}$ in the photosphere, sustained by radiative levitation, remains as unmistakable evidence that the star has undergone an episode of accretion. Our hypothesis is corroborated by the fact that no white dwarfs with close-in dusty disks have been found at $T_{\text {eff }} \gtrsim 23000 \mathrm{~K}$ (Farihi et al. 2009; Steele et al. 2011; Girven et al. 2011, 2012; Xu et al. 2013b; Barber et al. 2012) $)^{5}$.

In summary, at least $27 \%$ of the white dwarfs in our sample are currently accreting, and another $29 \%$ have photospheric metals sustained by radiative levitation. In the vast majority of the stars where both $\mathrm{Si}$ and $\mathrm{C}$ are detected, the $\mathrm{C} / \mathrm{Si}$ ratio suggests planetary material as the origin of the material. For the accreting white dwarfs, this is the only plausible origin as interstellar accretion cannot provide the observed accretion rates. Also taking the distribution of accreting white dwarfs into account, along with those where radiative levitation is sufficient to explain the detected metals, strongly suggests that the majority of all the metal-polluted stars in our COS sample are accreting, or have accreted planetary debris.

\footnotetext{
5 The hottest white dwarf hosting a close-in dusty disk is WD $0843+516$, with $T_{\text {eff }}=22412 \pm 304 \mathrm{~K}$ (Table 1, see also Gänsicke et al. 2012 and Xu \& Jura 2012). For completeness we note that infrared excesses of $T \approx 100 \mathrm{~K}$ material have been detected around several hot and young white dwarfs that are central stars of planetary nebulae $(\mathrm{Su}$ et al. 2007; Chu et al. 2009, 2011; Bilíková et al. 2012). However this material is inferred to lie at much larger separations from the star, at many tens of $\mathrm{AU}$ and its nature is still uncertain.
}

\subsection{Dependence on the mass of the white dwarfs and their progenitors}

The vast majority of exo-planet searches have focused on FGK type host stars, corresponding to a very narrow range in stellar masses, $\approx 0.5-1.5 M_{\odot}{ }^{6}$. Consequently, our current knowledge of the frequency, and architecture of planet hosts with $M>1.5 M_{\odot}$ is limited.

White dwarfs, on the other hand, are the burnt-out cores of stars with initial masses in the range $\approx 0.8-8 M_{\odot}$, and the progenitors of the majority of the present-day galactic white dwarf population had $\approx 2 M_{\odot}$. The white dwarf mass relates to the mass of its progenitor via the initial-mass to final-mass relation (Weidemann 1984, 2000; Catalán et al. 2008; Kalirai et al. 2008; Williams et al. 2009; Dobbie et al. 2009). The distribution of the progenitor masses of the 85 DA white dwarfs in our sample is shown in Fig. 1, illustrating that the median progenitor of our sample had indeed $\approx 2 M_{\odot}$.

The detection of debris-pollution hence probes the existence of planetary bodies around main sequence stars spanning a wide range of masses. More than half of the 23 stars in our sample that are currently accreting have estimated progenitor masses in excess of $2 M_{\odot}$, including the two Hyades white dwarfs discussed by Farihi et al. (2013b, see also Sect. 9.6) and WD 1015+161 which also exhibits infrared excess from circumstellar dust (Jura et al. 2007). This unmistakably demonstrates that the formation and existence of rocky planetary material around A-stars stars is common, and can survive the post-main sequence evolution of these stars. This is further strengthening the results of Bonsor et al. (2014) who detected cool dust with Herschel around 11\% of sub-giants with estimated masses in the range $1.5-1.8 M_{\odot}$ (see previous footnote).

Intriguing is an apparent lack of debris pollution for the highest mass white dwarfs, $M_{\mathrm{wd}}>0.8 M_{\odot}$, corresponding to progenitors with $M_{\mathrm{ms}} \gtrsim 3.8 M_{\odot}$ - we detected Si only in one out of 14 stars in this mass range, WD $1038+633$ with an estimated progenitor mass of $\approx 4.3 M_{\odot}$. Given that our COS sample is still relatively small, we investigated the possible effect of smallnumber statistics causing the observed distribution. A MonteCarlo simulation, randomizing the white dwarf masses in our sample, suggests that the lack of debris-polluted white dwarfs with $M_{\mathrm{wd}}>0.8 M_{\odot}$ being a chance result is $\$ 5 \%$; good enough to justify exploring possible causes for this deficiency.

The white dwarf mass distribution in Fig. 1 resembles closely that of larger well-studied samples (Finley et al. 1997; Liebert et al. 2005; Giammichele et al. 2012), displaying besides the dominant population around a mean mass of $0.6 M_{\odot}$ two additional peaks near $\approx 0.4 M_{\odot}$ and $\approx 0.8 M_{\odot}$. We come back to the low-mass peak below. Regarding the tail of highmass white dwarfs, general consensus is that their number is in excess to expectations from the galactic star formation history, and that a substantial number of them are the product of double white dwarf mergers. The complex, and in part violent evolution of such systems makes it unlikely that planetary bodies present around one, or both of the progenitors would survive, or that

\footnotetext{
6 Search for radial velocity variations and planetary transits becomes increasingly difficult with increasing mass: A-type stars have very few sharp features in their spectra that can be used for cross-correlation, and the depth of transits scales as $R_{\mathrm{p}}^{2} / R_{*}^{2}$, with $R_{\mathrm{p}}$ and $R_{*}$ the planet and host star radii. The former issue can be circumvented by observing "retired A-stars", i.e. subgiants with estimated $M>1.5 M_{\odot}$. While there have been a number of planet detections, there is an intense ongoing debate regarding the true masses of subgiant planet hosts (Bowler et al. 2010; Johnson et al. 2010, 2013; Lloyd 2013, 2011).
} 
long-lived circumstellar disks would be formed frequently, explaining the lack of debris-pollution among those stars.

Given that the main-sequence life time rapidly increases with decreasing stellar mass, the Galaxy is not old enough to have formed low mass white dwarfs $\left(M_{\mathrm{wd}} \leq 0.45 M_{\odot}\right)$ from single-star evolution. In other words, all low-mass white dwarfs must have undergone binary interactions that truncated their core-growth prior to the onset of He-burning, and consequently they have He-cores. A frequent pathway to He-core white dwarfs is the common envelope interaction with a mainsequence star, often of low mass (Rebassa-Mansergas et al. 2011). In fact, two of the post-common envelope binaries that we included in our survey as comparison objects (WD 0710+741, WD 2257+162) have He-core white dwarfs. Alternatively, two sufficiently massive main-sequence stars can evolve into close double-degenerate binaries, containing one, or two He-core white dwarfs. There is, however, also a small number of apparently single low-mass white dwarfs, and Nelemans \& Tauris (1998) suggested that massive planets may be sufficient to result in the ejection of the envelope of their host star.

Our COS sample includes five white dwarfs with masses $<0.45 M_{\odot}$, which we consider a conservative limit for containing He-cores. WD 0341+021 and WD 1713+332 show photospheric Si compatible with radiative levitation, whereas no metals are detected in WD 1229-013, WD 1353+409, and WD 2032+188. All five systems have been investigated for radial velocities, WD $1713+332$ and WD 2032+188 are confirmed doubledegenerate binaries with periods of $1.12 \mathrm{~d}$ and $5.08 \mathrm{~d}$ (Marsh et al. 1995; Nelemans et al. 2005), WD 0341+021 is a suspected double-degenerate (Maxted et al. 2000), and WD 1229-013 and WD 1353+409 show no radial velocity variations (Maxted \& Marsh 1998; Maxted et al. 2000). Given our arguments in Sect. 9.1, it hence appears that stellar wind accretion can occur onto white dwarfs in close binaries. One other known example of a very likely He-core that is metal-polluted and has a dusty debris disk is SDSSJ $155720.77+091624.7$, suggesting that complex planetary systems in close binary systems can form (Girven et al. 2011; Steele et al. 2011; Farihi et al. 2012a).

\subsection{The two Hyades white dwarfs}

Farihi et al. (2013b) interpreted the detection of photospheric Si in two Hyades white dwarfs, WD 0421+162 and WD 0431+126, as evidence for the presence of rocky planetesimals. The calculations of the diffusion fluxes in that study did not account for the effect of radiative levitation, which attracted some criticism from Chayer (2014). He finds that WD 0421+162 needs accretion to explain the observed Si, but that the WD 0431+126 can be explained by radiative levitation alone. While we agree on the first object, we disagree on the second. In our calculations accretion has to also be invoked for this object. In our model for zero accretion, we find indeed radiative support between optical depths $5.1 \times 10^{-6}$ to $3.2 \times 10^{-4}$ with a maximum Si abundance of $\log [\mathrm{Si} / \mathrm{H}]=-7.47$, and between 1.9 to 8.4 with a maximum of -7.72 (note that the parameters are similar to those of the model in Fig. 7). These numbers are very similar to Chayer's values in his Fig. 2. However, between these two "Si clouds" the abundance falls below -12.00, and the equivalent width for Si II 1265 calculated with this model is only $2 \mathrm{~m} \AA$, as compared to the observed $55 \mathrm{~m} \AA$. The reasons for the difference are discussed above; the main point here is that $\mathrm{C} 10$ do not calculate the abundance distribution within the visible photosphere. In any case, WD0431+126 is close to the limiting line of Fig. 5 and small differences in the models can shift the result either way.
In summary, including the effects of radiative levitation somewhat reduces the diffusion flux in WD $0431+126$ with respect to the value in Farihi et al. (2013b), however the discussion and conclusion of that paper remain unaffected.

\section{Conclusion}

We have carried out an ultraviolet high-resolution survey of 85 DA with $17000 \mathrm{~K}<T_{\text {eff }} \lesssim 27000 \mathrm{~K}$ white dwarfs with relatively short cooling ages (20-200 Myr), and we found photospheric metal pollution by at least $\mathrm{Si}$ in $52 \%$ of our target stars. The correct interpretation of the data required a full treatment of radiative levitation, which we have now implemented in our atmosphere code.

At least $27 \%$ of these stars are currently accreting material that is consistent with planetary origin, and an additional $29 \%$ have undergone at least one episode of accretion as we see $\mathrm{Si}$ and in some cases $\mathrm{C}$ that is sustained by radiative levitation. At temperatures $17000 \mathrm{~K}<T_{\text {eff }} \lesssim 20000 \mathrm{~K}$, the fraction of white dwarfs accreting planetary debris is $\approx 50 \%$. While we cannot exclude that accretion from the ISM is responsible for the metals in a few of the stars that are not currently accreting, their distribution in $T_{\text {eff }}$ strongly suggest that planetary debris is also the principle, if not sole, cause of their photospheric pollution. Hence, the fraction of white dwarfs accreting planetary debris is plausible close to $50 \%$, similar to the fraction of stars hosting super-Earths (Gaidos 2013).

At stellar temperatures $\gtrsim 23000 \mathrm{~K}$ the fraction of currently accreting white dwarfs rapidly drops, which is consistent with the fact that these stars are too hot to allow the formation of longlived dusty disks that are necessary to provide a continuous flow of metals. Tidal disruption of planetary bodies in these systems will lead to short-lived accretion events from purely gaseous disks, reducing the probability of catching these stars during this phase - yet, radiative levitation provides the memory to past accretion. One prediction of this hypothesis is that a small number of warm DA stars should be found to be accreting at much higher rates than the cooler stars with dusty disks. We note that photospheric and circumstellar metals are detected in a number of white dwarfs that are much hotter, and much younger than our sample. The origin of the metals has been intensively debated in the past (Shipman et al. 1995; Bannister et al. 2003; Barstow et al. 2003; Dickinson et al. 2012a,b), but it is very likely that it is, at least in part, also related to planetary debris (Barstow et al. 2014). One particularly promising candidate of a hot white dwarf accreting planetary debris at a high rate is the extremely metal-polluted GD394 ( $\approx 40000$ K, Barstow et al. 1996; Dupuis et al. 2000).

We find that about half of the white dwarfs that are currently accreting planetary debris have progenitor masses of 2-3 $M_{\odot}$ corresponding to late B- and A-type stars, which shows that the formation of rocky planetary material is common around mainsequence stars in this mass range. At the highest white dwarf masses, $M_{\mathrm{wd}}>0.8 M_{\odot}$, metal pollution is extremely uncommon, consistent with the hypothesis that most of these massive white dwarfs are the product of double-degenerate mergers, instead of single-star evolution.

Finally, combining published ground-based searches for metal-pollution in white dwarfs with the results from our COS survey, we find that neither the fraction of accreting white dwarfs, nor the rates at which they accrete, show a noticeable correlation over an extremely wide range of cooling ages, $\approx 20 \mathrm{Myr}-2$ Gyr. This broadly confirms the evolutionary simulations of Veras et al. (2013) who found that instabilities in the 
remnants of two-planet systems are likely to occur over roughly the same period in time.

Acknowledgements. Based on observations made with the NASA/ESA Hubble Space Telescope, obtained at the Space Telescope Science Institute, which is operated by the Association of Universities for Research in Astronomy, Inc., under NASA contract NAS 5-26555. These observations are associated with program $\# 12169$ and \#12474. The research leading to these results has received funding from the European Research Council under the European Union's Seventh Framework Programme (FP/2007-2013)/ERC Grant Agreement n. 320964 (WDTracer). B.T.G. was supported in part by the UKs Science and Technology Facilities Council (ST/I001719/1). Balmer lines in the models were calculated with the modified Stark broadening profiles of Tremblay \& Bergeron (2009) kindly made available by the authors. This research was made possible through the use of the AAVSO Photometric All-Sky Survey (APASS), funded by the Robert Martin Ayers Sciences Fund.

\section{References}

Aannestad, P. A., Kenyon, S. J., Hammond, G. L., \& Sion, E. M. 1993, AJ, 105, 1033

Alcock, C., \& Illarionov, A. 1980, ApJ, 235, 541

Allard, N., \& Kielkopf, J. 1982, Rev. Mod. Phys., 54, 1103

Allard, N. F., Royer, A., Kielkopf, J. F., \& Feautrier, N. 1999, Phys. Rev. A, 60, 1021

Asplund, M., Grevesse, N., Sauval, A. J., \& Scott, P. 2009, ARA\&A, 47, 481

Bannister, N. P., Barstow, M. A., Holberg, J. B., \& Bruhweiler, F. C. 2003, MNRAS, 341, 477

Barber, S. D., Patterson, A. J., Kilic, M., et al. 2012, ApJ, 760, 26

Barstow, M. A., Holberg, J. B., Hubeny, I., et al. 1996, MNRAS, 279, 1120

Barstow, M. A., Good, S. A., Holberg, J. B., et al. 2003, MNRAS, 341, 870

Barstow, M. A., Barstow, J. K., Casewell, S. L., Holberg, J. B., \& Hubeny, I. 2014, MNRAS, 440, 1607

Becklin, E. E., Farihi, J., Jura, M., et al. 2005, ApJ, 632, L119

Berger, L., Koester, D., Napiwotzki, R., Reid, I. N., \& Zuckerman, B. 2005, A\&A, 444, 565

Bergeron, P., Wesemael, F., Dufour, P., et al. 2011, ApJ, 737, 28

Bilíková, J., Chu, Y.-H., Gruendl, R. A., Su, K. Y. L., \& De Marco, O. 2012, ApJS, 200, 3

Bonsor, A., \& Wyatt, M. C. 2011, in AIP Conf. Ser. 1331, eds. S. Schuh, H. Drechsel, \& U. Heber, 41

Bonsor, A., Mustill, A. J., \& Wyatt, M. C. 2011, MNRAS, 414, 930

Bonsor, A., Kennedy, G. M., Wyatt, M. C., Johnson, J. A., \& Sibthorpe, B. 2014, MNRAS, 437, 3288

Bowler, B. P., Johnson, J. A., Marcy, G. W., et al. 2010, ApJ, 709, 396 Brinkworth, C. S., Gänsicke, B. T., Girven, J. M., et al. 2012, ApJ, 750, 86 Cassan, A., Kubas, D., Beaulieu, J.-P., et al. 2012, Nature, 481, 167

Catalán, S., Isern, J., García-Berro, E., \& Ribas, I. 2008, MNRAS, 387, 1693

Chayer, P. 2014, MNRAS, 437, L95

Chayer, P., \& Dupuis, J. 2010, in AIP Conf. Ser. 1273, eds. K. Werner, \&

T. Rauch, 394

Chayer, P., Fontaine, G., \& Wesemael, F. 1995a, ApJS, 99, 189

Chayer, P., Vennes, S., Pradhan, A. K., et al. 1995b, ApJ, 454, 429

Chu, Y.-H., Gruendl, R. A., Guerrero, M. A., et al. 2009, AJ, 138, 691

Chu, Y.-H., Su, K. Y. L., Bilikova, J., et al. 2011, AJ, 142, 75

Davidsson, B. J. R. 1999, Icarus, 142, 525

Deal, M., Deheuvels, S., Vauclair, G., Vauclair, S., \& Wachlin, F. C. 2013, A\&A, 557, L12

Debes, J. H., \& Sigurdsson, S. 2002, ApJ, 572, 556

Debes, J. H., Walsh, K. J., \& Stark, C. 2012, ApJ, 747, 148

Dickinson, N. J., Barstow, M. A., \& Hubeny, I. 2012a, MNRAS, 421, 3222

Dickinson, N. J., Barstow, M. A., Welsh, B. Y., et al. 2012b, MNRAS, 423, 1397

Dobbie, P. D., Napiwotzki, R., Burleigh, M. R., et al. 2009, MNRAS, 395, 2248

Dreizler, S., \& Wolff, B. 1999, A\&A, 348, 189

Dufour, P., Kilic, M., Fontaine, G., et al. 2010, ApJ, 719, 803

Dufour, P., Kilic, M., Fontaine, G., et al. 2012, ApJ, 749, 6

Duncan, M. J., \& Lissauer, J. J. 1998, Icarus, 134, 303

Dupuis, J., Fontaine, G., Pelletier, C., \& Wesemael, F. 1992, ApJS, 82, 505

Dupuis, J., Chayer, P., Vennes, S., Christian, D. J., \& Kruk, J. W. 2000, ApJ, 537, 977

Dupuis, J., Chayer, P., \& Hénault-Brunet, V. 2010, in AIP Conf. Ser. 1273, eds K. Werner, \& T. Rauch, 412

Farihi, J. 2011, White Dwarf Circumstellar Disks: Observations (Berlin: Whiley-VCH), 117

Farihi, J., Zuckerman, B., \& Becklin, E. E. 2008, ApJ, 674, 431

Farihi, J., Jura, M., \& Zuckerman, B. 2009, ApJ, 694, 805
Farihi, J., Barstow, M. A., Redfield, S., Dufour, P., \& Hambly, N. C. 2010a, MNRAS, 404, 2123

Farihi, J., Hoard, D. W., \& Wachter, S. 2010b, ApJS, 190, 275

Farihi, J., Gänsicke, B. T., Steele, P. R., et al. 2012a, MNRAS, 421, 1635

Farihi, J., Gänsicke, B. T., Wyatt, M. C., et al. 2012b, MNRAS, 424, 464

Farihi, J., Gänsicke, B. T., \& Koester, D. 2013a, Science, 342, 218

Farihi, J., Gänsicke, B. T., \& Koester, D. 2013b, MNRAS, 432, 1955

Finley, D., Koester, D., Kruk, J., Kimble, R., \& Allard, N. 1997, in White Dwarfs, Proc. 10th European Workshop on White Dwarfs, eds. J. Isern, M. Hernanz, \& E. Garcia-Berro, 245

Fontaine, G., \& Michaud, G. 1979, ApJ, 231, 826

Fontaine, G., Villeneuve, B., Wesemael, F., \& Wegner, G. 1984, ApJ, 277, L61

Fontaine, G., Brassard, P., \& Bergeron, P. 2001, PASP, 113, 409

Fressin, F., Torres, G., Charbonneau, D., et al. 2013, ApJ, 766, 81

Frewen, S. F. N., \& Hansen, B. M. S. 2014, MNRAS, 439, 2442

Friedrich, S., Jordan, S., \& Koester, D. 2004, A\&A, 424, 665

Gaidos, E. 2013, ApJ, 770, 90

Gänsicke, B. T., Marsh, T. R., Southworth, J., \& Rebassa-Mansergas, A. 2006, Science, 314, 1908

Gänsicke, B. T., Marsh, T. R., \& Southworth, J. 2007, MNRAS, 380, L35

Gänsicke, B. T., Koester, D., Marsh, T. R., Rebassa-Mansergas, A., \& Southworth, J. 2008, MNRAS, 391, L103

Gänsicke, B. T., Koester, D., Farihi, J., et al. 2012, MNRAS, 424, 333

Giammichele, N., Bergeron, P., \& Dufour, P. 2012, ApJS, 199, 29

Gianninas, A., Bergeron, P., \& Ruiz, M. T. 2011, ApJ, 743, 138

Girven, J., Gänsicke, B. T., Steeghs, D., \& Koester, D. 2011, MNRAS, 417, 1210

Girven, J., Brinkworth, C. S., Farihi, J., et al. 2012, ApJ, 749, 154

Gonzalez, J.-F., LeBlanc, F., Artru, M.-C., \& Michaud, G. 1995, A\&A, 297, 223

Graham, J. R., Matthews, K., Neugebauer, G., \& Soifer, B. T. 1990, ApJ, 357, 216

Green, J. C., Froning, C. S., Osterman, S., et al. 2012, ApJ, 744, 60

Hartmann, S., Nagel, T., Rauch, T., \& Werner, K. 2011, A\&A, 530, A7

Johnson, J. A., Aller, K. M., Howard, A. W., \& Crepp, J. R. 2010, PASP, 122, 905

Johnson, J. A., Morton, T. D., \& Wright, J. T. 2013, ApJ, 763, 53

Jura, M. 2003, ApJ, 584, L91

Jura, M. 2008, AJ, 135, 1785

Jura, M., Farihi, J., \& Zuckerman, B. 2007, ApJ, 663, 1285

Jura, M., Xu, S., Klein, B., Koester, D., \& Zuckerman, B. 2012, ApJ, 750, 69

Kalirai, J. S., Hansen, B. M. S., Kelson, D. D., et al. 2008, ApJ, 676, 594

Kilic, M., Patterson, A. J., Barber, S., Leggett, S. K., \& Dufour, P. 2012, MNRAS, 419, L59

Klein, B., Jura, M., Koester, D., Zuckerman, B., \& Melis, C. 2010, ApJ, 709, 950

Klein, B., Jura, M., Koester, D., \& Zuckerman, B. 2011, ApJ, 741, 64

Koester, D. 2009, A\&A, 498, 517

Koester, D. 2010, Mem. Sos. Astron. It., 81, 921

Koester, D., \& Wilken, D. 2006, A\&A, 453, 1051

Koester, D., Weidemann, V., \& Zeidler-KT, E. M. 1982, A\&A, 116, 147

Koester, D., Rollenhagen, K., Napiwotzki, R., et al. 2005, A\&A, 432, 1025

Koester, D., Voss, B., Napiwotzki, R., et al. 2009, A\&A, 505, 441

Liebert, J., Bergeron, P., \& Holberg, J. B. 2005, ApJS, 156, 47

Lloyd, J. P. 2011, ApJ, 739, L49

Lloyd, J. P. 2013, ApJ, 774, L2

Marsh, T. R., Dhillon, V. S., \& Duck, S. R. 1995, MNRAS, 275, 828

Maxted, P. F. L., \& Marsh, T. R. 1998, MNRAS, 296, L34

Maxted, P. F. L., Marsh, T. R., \& North, R. C. 2000, MNRAS, 317, L41

McDonough, W. 2000, in Earthquake Thermodynamics and Phase Transformation in the Earth's Interior, eds. R. Teisseyre, \& E. Majewski (Elsevier Science Academic Press), 5

Melis, C., Dufour, P., Farihi, J., et al. 2012, ApJ, 751, L4

Metzger, B. D., Rafikov, R. R., \& Bochkarev, K. V. 2012, MNRAS, 423, 505

Michaud, G., Martel, A., Montmerle, T., et al. 1979, ApJ, 234, 206

Mustill, A. J., \& Villaver, E. 2012, ApJ, 761, 121

Napiwotzki, R., Christlieb, N., Drechsel, H., et al. 2001, Astron. Nachr., 322, 411

Nelemans, G., \& Tauris, T. M. 1998, A\&A, 335, L85

Nelemans, G., Napiwotzki, R., Karl, C., et al. 2005, A\&A, 440, 1087

Nordhaus, J., Spiegel, D. S., Ibgui, L., Goodman, J., \& Burrows, A. 2010, MNRAS, 408, 631

Paquette, C., Pelletier, C., Fontaine, G., \& Michaud, G. 1986, ApJS, 61, 177

Pelletier, C., Fontaine, G., Wesemael, F., Michaud, G., \& Wegner, G. 1986, ApJ, 307, 242

Rafikov, R. R. 2011a, ApJ, 732, L3

Rafikov, R. R. 2011b, MNRAS, 416, L55

Rafikov, R. R., \& Garmilla, J. A. 2012, ApJ, 760, 123

Rebassa-Mansergas, A., Nebot Gómez-Morán, A., Schreiber, M. R., Girven, J., \& Gänsicke, B. T. 2011, MNRAS, 413, 1121 
Redfield, S., \& Linsky, J. L. 2004, ApJ, 602, 776

Sackmann, I. J., Boothroyd, A. I., \& Kraemer, K. E. 1993, ApJ, 418, 457

Santos, M. G., \& Kepler, S. O. 2012, MNRAS, 423, 68

Savage, B. D., \& Sembach, K. R. 1996, ARA\&A, 34, 279

Schatzman, E. 1947, Ann. Astrophys., 10, 19

Schreiber, M. R., \& Gänsicke, B. T. 2003, A\&A, 406, 305

Shipman, H. L., Provencal, J., Roby, S. W., et al. 1995, AJ, 109, 1220

Steele, P. R., Burleigh, M. R., Dobbie, P. D., et al. 2011, MNRAS, 416, 2768

Su, K. Y. L., Chu, Y., Rieke, G. H., et al. 2007, ApJ, 657, L41

Tremblay, P.-E., \& Bergeron, P. 2009, ApJ, 696, 1755

Vauclair, G., Vauclair, S., \& Greenstein, J. L. 1979, A\&A, 80, 79

Vennes, S., Pelletier, C., Fontaine, G., \& Wesemael, F. 1988, ApJ, 331, 876

Vennes, S., Kawka, A., \& Németh, P. 2010, MNRAS, 404, L40

Vennes, S., Kawka, A., \& Németh, P. 2011a, MNRAS, 413, 2545

Vennes, S., Thorstensen, J. R., Kawka, A., et al. 2011b, ApJ, 737, L16

Veras, D., Wyatt, M. C., Mustill, A. J., Bonsor, A., \& Eldridge, J. J. 2011 MNRAS, 417, 2104

Veras, D., Mustill, A. J., Bonsor, A., \& Wyatt, M. C. 2013, MNRAS, 431, 1686

Villaver, E., \& Livio, M. 2007, ApJ, 661, 1192

Villaver, E., \& Livio, M. 2009, ApJ, 705, L81 von Hippel, T., Kuchner, M. J., Kilic, M., Mullally, F., \& Reach, W. T. 2007, ApJ, 662, 544

Voyatzis, G., Hadjidemetriou, J. D., Veras, D., \& Varvoglis, H. 2013, MNRAS 430, 3383

Weidemann, V. 1984, in Observational Tests of the Stellar Evolution Theory, IAU Symp., 105, 229

Weidemann, V. 2000, A\&A, 363, 647

Williams, K. A., Bolte, M., \& Koester, D. 2009, ApJ, 693, 355

Wolff, B., Koester, D., \& Liebert, J. 2002, A\&A, 385, 995

Xu, S., \& Jura, M. 2012, ApJ, 745, 88

Xu, S., Jura, M., Klein, B., Koester, D., \& Zuckerman, B. 2013a, ApJ, 766, 132

Xu, S., Jura, M., Koester, D., Klein, B., \& Zuckerman, B. 2013b, ApJ, 766, L18

Xu, S., Jura, M., Koester, D., Klein, B., \& Zuckerman, B. 2014, ApJ, 783, 79

Zuckerman, B., \& Becklin, E. E. 1987, ApJ, 319, L99

Zuckerman, B., Koester, D., Reid, I. N., \& Hünsch, M. 2003, ApJ, 596, 477

Zuckerman, B., Koester, D., Melis, C., Hansen, B. M., \& Jura, M. 2007, ApJ, 671,872

Zuckerman, B., Melis, C., Klein, B., Koester, D., \& Jura, M. 2010, ApJ, 722, 725

Zuckerman, B., Koester, D., Dufour, P., et al. 2011, ApJ, 739, 101

Zuckerman, B., Xu, S., Klein, B., \& Jura, M. 2013, ApJ, 770, 140 


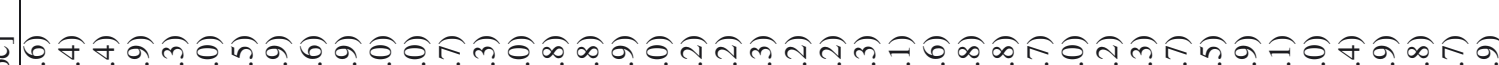
ق

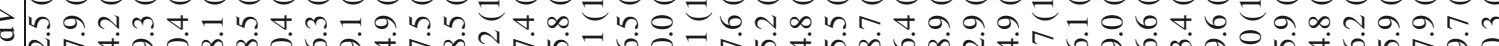

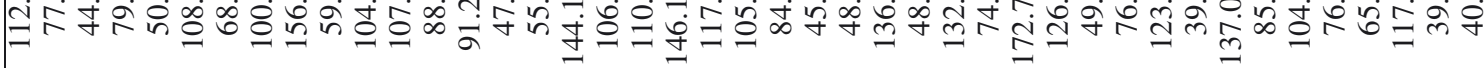

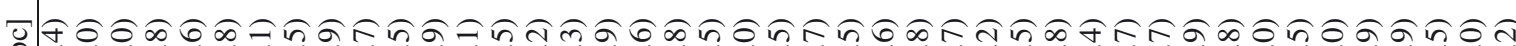
ó 馬

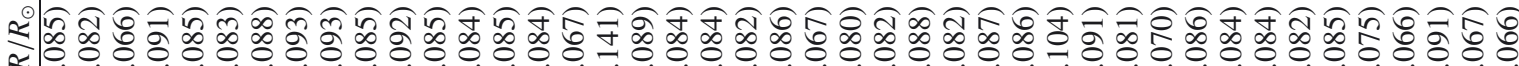

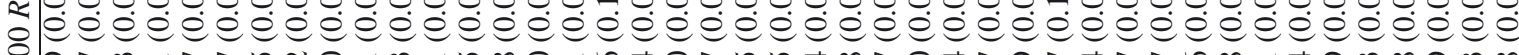

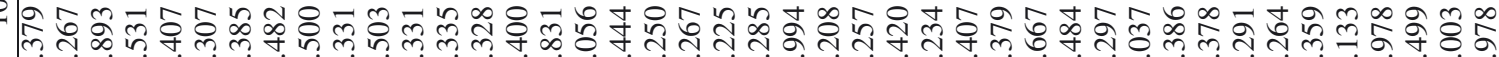

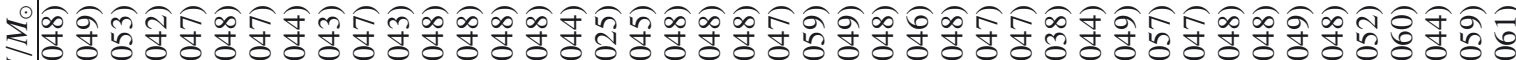

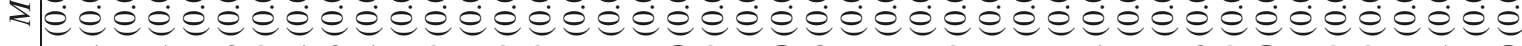
궁 o :

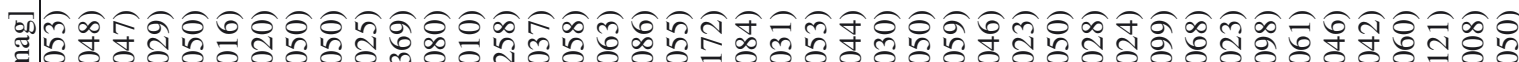

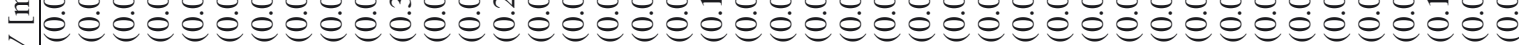

๓

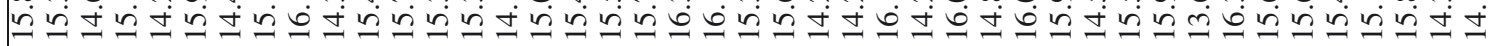

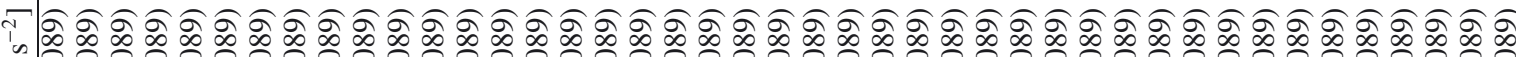

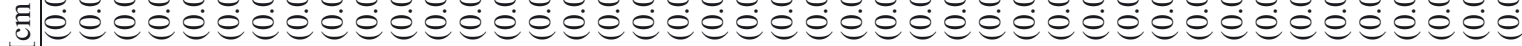

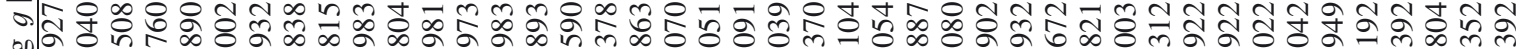

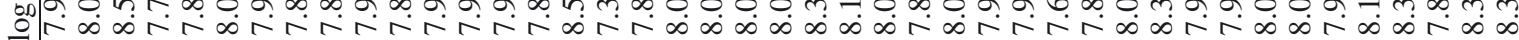

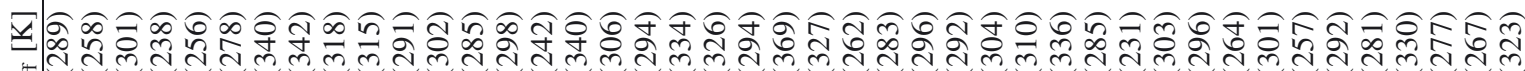

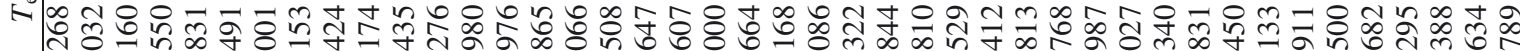

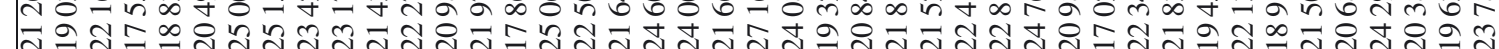

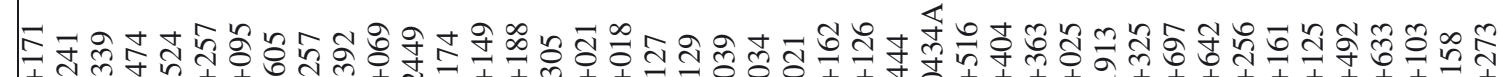

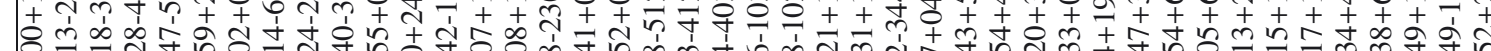

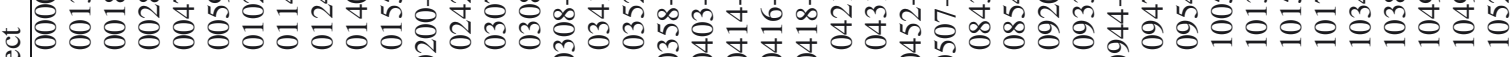

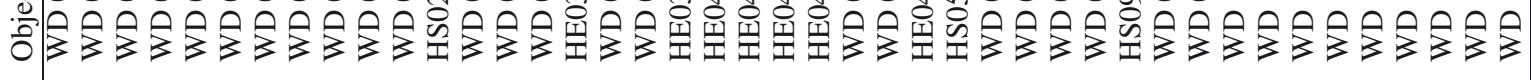




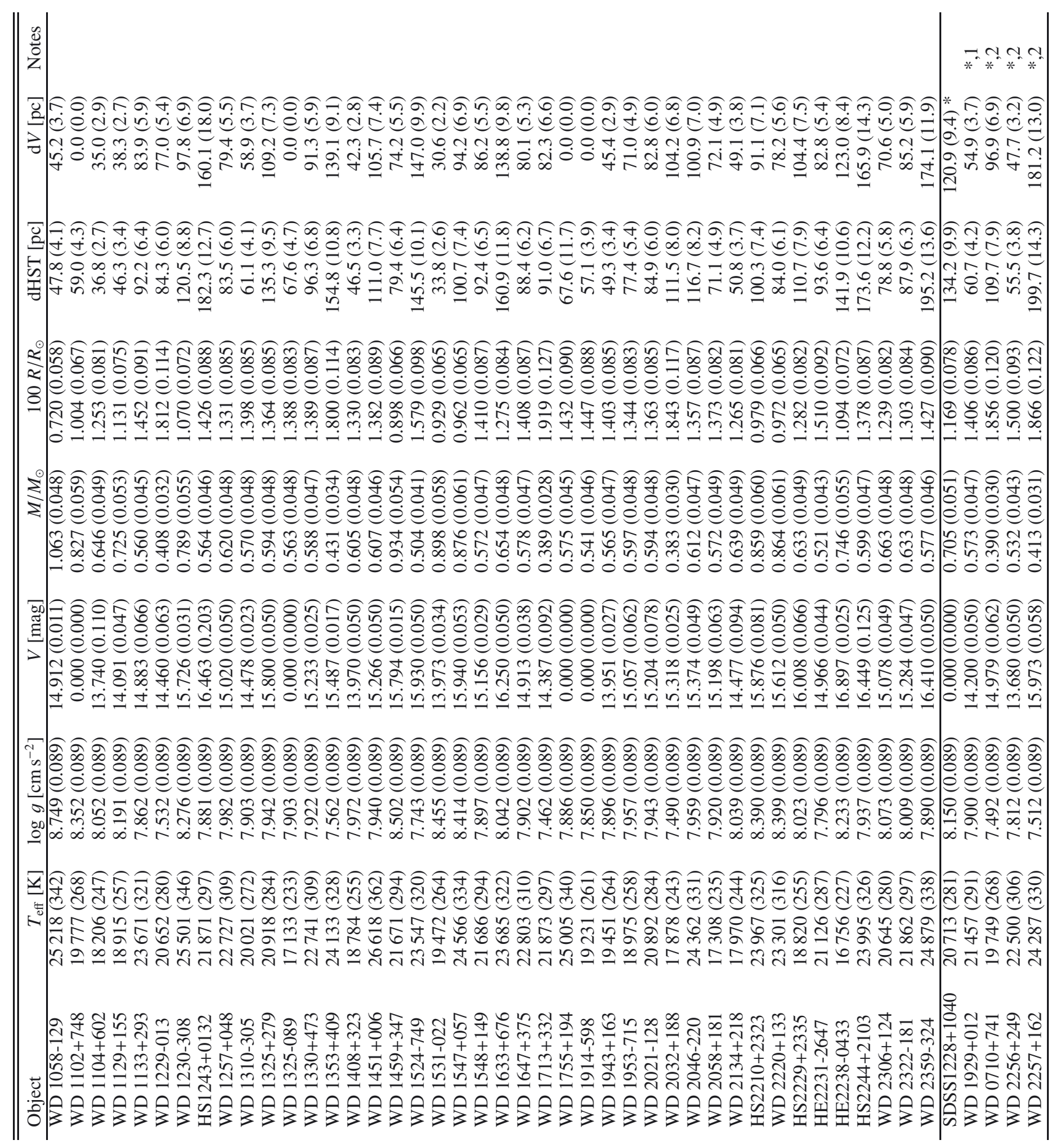

